\title{
Estimation of Preference Heterogeneity in Markets with Costly Search*
}

\author{
Ilya Morozov Stephan Seiler Xiaojing Dong Liwen Hou \\ Northwestern Imperial Santa Clara Shanghai Jiao \\ University College University Tong University
}

This draft: November 18, 2020

\begin{abstract}
We study the estimation of preference heterogeneity in markets where consumers engage in costly search to learn product characteristics. Costly search amplifies the way consumer preferences translate into purchase probabilities, generating a seemingly large degree of preference heterogeneity. We develop a search model that allows for flexible preference heterogeneity and estimate its parameters using a unique panel dataset on the search and purchase behavior of consumers. The results reveal that when search costs are ignored, the model overestimates standard deviations of product intercepts by $53 \%$. We show that the bias in heterogeneity estimates leads to incorrect inference about price elasticities and seller markups and has important consequences for personalized pricing.
\end{abstract}

Keywords: Consumer Search, Preference Heterogeneity, Choice Persistence, Importance Sampling

*We thank Bart Bronnenberg, Øystein Daljord, Robert Donnelly, Wes Hartmann, Elisabeth Honka, Suraj Malladi, Andrew Rhodes, and Peter Rossi for helpful comments. We also thank seminar participants at Carnegie Mellon, Frankfurt University, Georgia Tech, Santa Clara, UCLA, and Wharton as well as conference participants at Marketing Dynamics 2018, Summer Institute for Competitive Strategy 2018, and the Winter Marketing-Economics Summit 2018. All errors are our own. 


\section{Introduction}

One of the most well-known and widely studied stylized facts in the marketing literature is that consumers exhibit strong persistence in product choices (Rossi and Allenby, 1993; Rossi, McCulloch, and Allenby, 1996; Allenby and Rossi, 1999). Researchers often interpret this persistence as evidence that consumers have heterogeneous preferences over existing products. In this paper, we challenge the conventional wisdom that strong persistence in choices is necessarily driven by strong preference heterogeneity. We argue that in the presence of search frictions, even mild preferences over products can translate into highly persistent choices. ${ }^{1}$

The contribution of this paper is twofold. First, we demonstrate how preference heterogeneity and search frictions jointly determine persistence in consumer choices. When search is costly, consumers first search their most preferred product and are less likely to evaluate other options. This search behavior concentrates purchase probabilities around each consumer's preferred product. A researcher who ignores search frictions may incorrectly infer that consumers strongly prefer certain products, whereas in reality, slight preferences across products are amplified by the presence of search frictions. This amplification is especially important in the light of the recent research that finds substantial search costs in a broad range of product categories (De Los Santos, Hortacsu, and Wildenbeest, 2012; Seiler, 2013; Honka, 2014; Koulayev, 2014; Giulietti, Waterson, and Wildenbeest, 2014). As a result, preference heterogeneity in many markets may be less pronounced than previously thought. ${ }^{2}$

Second, we show that accounting for search frictions is quantitatively important. To illustrate this point, we estimate a search model with flexible preference heterogeneity using search and purchase data from a large online retailer. The results reveal that ignoring search frictions leads to a $53 \%$ upward bias in the estimated preference heterogeneity, measured by the standard deviations of product intercepts. Since this bias implies a seemingly large degree of product differentiation, we underestimate absolute values of own-price elasticities by $40 \%$ and overestimate the markups of firms by $19 \%$. Furthermore, because consumers appear more heterogeneous in our estimates than they actually are, we also overestimate the scope for personalized pricing. Optimal personalized prices are more dispersed in a perfect-information model than in a model that explicitly accounts for search frictions. This difference in personalized prices affects the expected profit from personalization: while pricing based on the search model increases profits by $9.1 \%$, pricing based on the perfect information model actually reduces profits by $0.6 \%$. Hence, accounting for search frictions is crucial for evaluating the degree of competition among sellers and for implementing optimal personalized pricing.

We estimate our model using a unique panel dataset on consumer search and purchase behavior. We observe each consumer visiting the retailer's website multiple times, and our dataset describes

\footnotetext{
${ }^{1}$ Switching costs provide another possible explanation for choice persistence (Dubé, Hitsch, Rossi, and Vitorino, 2008; Dubé, Hitsch, and Rossi, 2009). We discuss the role of switching costs in more detail at the end of this section.

${ }^{2}$ This conclusion may change when search costs substantially vary across products, in which case ignoring search may lead to underestimation of preference heterogeneity. In our application, however, we find that preference heterogeneity is overestimated even when we allow for different search cost across products.
} 
search and purchase behavior during each of these visits. This panel dimension is particularly useful in our setting because of several reasons. Since preference heterogeneity leads to persistence in choices over time, observing consumers repeatedly helps us estimate preference heterogeneity. We explicitly build our identification argument on this panel structure and show that with a sufficiently long panel, we can nonparametrically identify how preferences and search costs are distributed across consumers. Moreover, the panel dimension of the data allows us to analyze choice persistence and study how this persistence is jointly driven by preferences and search costs. Finally, we use the panel structure of the data to implement a robustness check that allows for correlation in information between consecutive search sessions.

Estimating our model is computationally burdensome because we have to integrate consumer heterogeneity out of the likelihood function, which is by itself difficult to compute. To reduce the computational burden, we implement an importance sampling procedure similar to that proposed by Ackerberg (2009). This approach removes the need to recompute individual likelihood contributions for each guess of parameters, thus substantially reducing computational costs. We show that using this approach makes estimation significantly faster than alternative methods and can therefore prove useful to other researchers who want to estimate flexible models of consumer search.

This paper connects two strands of literature. On the one hand, our model of sequential search is similar to other structural models from the consumer search literature (Kim, Albuquerque, and Bronnenberg, 2010; De Los Santos, Hortacsu, and Wildenbeest, 2012; Honka, 2014; Honka and Chintagunta, 2017; Chen and Yao, 2017). However, these papers estimate search models using cross-sectional data and do not focus on estimating preference heterogeneity. On the other hand, there exists a rich marketing literature that focuses on the estimation and identification of preference heterogeneity but assumes that consumers are perfectly informed about existing products and their characteristics (Chintagunta, Jain, and Vilcassim, 1991; Rossi and Allenby, 1993; Rossi, McCulloch, and Allenby, 1996; Allenby and Rossi, 1999). We connect these two areas of research by reexamining the estimation of preference heterogeneity in markets with costly search.

Our paper is also related to the broader literature on explaining persistence in consumer choices. Prior research suggests that choice persistence can be rationalized by the presence of preference heterogeneity (see papers cited above) or switching costs (Dubé, Hitsch, Rossi, and Vitorino, 2008; Dubé, Hitsch, and Rossi, 2009). We contribute to this literature by showing that search costs may also generate choice persistence. We show that, while the presence of search costs does not by itself generate persistence, search costs amplify the way preference heterogeneity translates into persistent choices. To simplify exposition and estimation, we abstract away from switching costs. Prior research suggests that switching costs play a modest role in shaping consumer choices relative to search costs (Honka, 2014) or heterogeneity in preferences (Dubé, Hitsch, and Rossi, 2010). ${ }^{3}$

The rest of the paper proceeds as follows. In sections 2 and 3, we develop a search model and

\footnotetext{
${ }^{3}$ Honka (2014) finds that removing search costs from her estimated model has a 17-times larger effect on the persistence in consumers' choices than removing switching costs. We also expect the role of switching costs to be relatively less important in our context of consumer goods relative to the car insurance market studied in Honka (2014).
} 
analyze the impact of search costs on consumers' purchase decisions, choice persistence, and estimated preference heterogeneity. We then adapt this search model to accommodate panel data and discuss the identification of model's parameters in section 4. We describe our estimation strategy in section 5. Section 6 presents the estimation results, whereas section 7 analyzes implications for personalized pricing. We offer concluding remarks in section 8.

\section{General Model Framework}

Suppose a consumer $i$ conducts $T_{i}$ search sessions which we index by $t=1, \ldots, T_{i}$. In each session, she chooses exactly one product out of $J$ available alternatives. The utility consumer $i$ derives from product $j$ in session $t$ is the sum of a consumer-specific product intercept and a taste shock:

$$
u_{i j t}=\xi_{i j}+\varepsilon_{i j t} .
$$

We assume the consumer is imperfectly informed about product-specific utilities and must engage in costly search to resolve uncertainty. In particular, she knows product intercepts $\xi_{i j}$ and the distribution $F_{\varepsilon}(\varepsilon)$ from which the i.i.d. taste shocks $\varepsilon_{i j t}$ are drawn, but she must search to learn realizations of these taste shocks. The taste shocks are i.i.d. across consumers, products, and search sessions. ${ }^{4}$ There is no outside option, so the consumer always chooses one of the available options. ${ }^{5}$

Following Weitzman (1979), we assume the consumer searches sequentially and incurs a cost $c_{i j}$ for each searched product. Upon finishing search, she chooses one product from the searched options. In this setting, the optimal search behavior can be characterized by a simple threshold rule derived in Weitzman's paper. Define reservation utility of consumer $i$ for product $j$ as the unique value $z_{i j}$ that solves the equation ${ }^{6}$

$$
\int_{z_{i j}}^{\infty}\left(u_{i j t}-z_{i j}\right) d F\left(u_{i j t}\right)=c_{i j} .
$$

That is, the reservation utility $z_{i j}$ is the level of utility at which the consumer is indifferent between

\footnotetext{
${ }^{4}$ When introducing our empirical model in secton 4, we expand this framework by adding another taste shock known prior to search and adding price to the utility function.

${ }^{5}$ In our empirical application, we define search sessions retrospectively as all search activity within a week prior to a purchase. Since it is more difficult to define search sessions for consumers without purchases, we omit them from the analysis and assume that all consumers have to purchase one of the available options, thus removing the outside option. The search rules derived in this section would be similar in the presence of an outside option. One can simply think of the outside option as an additional product in the choice set that is always available to consumers (i.e. no search cost needs to be incurred). The search order condition would remain the same. The stopping rule maintains the same general structure, but the maximum realized utility of searched option now includes the outside option. Similar, the purchase decision is based on the maximum utility from all searched options, including the outside option. In our empirical application, we define search sessions retrospectively as all search activity that occurred within a week prior to a purchase. Since it is significantly more difficult to define search sessions for consumers without purchases, we omit them from the analysis and assume that all consumers have to purchase one of the available options, thus removing the outside option.

${ }^{6}$ We assume that the distribution of $\varepsilon_{i j t}$ is continuous and has full support, which ensures that the solution to this equation exists and is unique for any value of $c_{i j}$.
} 
searching product $j$ and receiving $z_{i j}$ with certainty. ${ }^{7}$ In the optimum, the consumer searches products in order of descending reservation utilities. At each step during this process, she continues searching as long as the maximum realized utility is lower than the reservation utility of the next product in the search sequence; otherwise, she stops searching and purchases the highest-utility product among the searched options.

\section{Preference Heterogeneity and Search Costs}

In this section we describe how preference heterogeneity and search costs jointly drive consumer choices. Additionally, we show that search costs increase choice persistence and demonstrate how ignoring search costs may bias the estimates of preference heterogeneity. We first build intuition using a simple example with two consumers and two products and then show that our conclusions also apply to a more general setting.

\subsection{Simple Example}

Consider a market with two consumers (1 and 2) choosing between two products (A and B). We normalize product intercepts for product $\mathrm{A}$ to zero (i.e. $\xi_{1 \mathrm{~A}}=\xi_{2 A}=0$ ) and assume consumer 1 prefers product $\mathrm{A}$ to product $\mathrm{B}\left(\xi_{1 B}=-1\right)$, whereas consumer 2 prefers product $\mathrm{B}$ to product $\mathrm{A}$ $\left(\xi_{2 B}=1\right)$. The taste shocks $\varepsilon_{i j t}$ are i.i.d. standard normal.

Our goal is to compare the purchase behavior of consumers under two scenarios: when they have perfect information about products, and when they engage in costly search. We start by considering the perfect-information scenario. In this scenario, consumer 1 purchases product A with probability

$$
\operatorname{PurchProb}_{1 A}(c=0)=\operatorname{Pr}\left(0+\varepsilon_{i A t} \geq-1+\varepsilon_{i B t}\right)=0.76,
$$

which corresponds to a standard probit purchase probability. We compute purchase probabilities for the other three consumer-product pairs in a similar way and report the results in Panel A of Table 1. According to the computed values, each consumer purchases the product with the higher intercept with probability $76 \%$. The probability of purchasing the same product in consecutive time periods is then equal to $64 \%$.

Next, we consider a second scenario in which consumers engage in costly search and face a search cost of $c=0.5$. Unlike in the perfect-information scenario, consumers now actively decide which products they want to search and in which order they want to search them. For example, according to the optimal search rule, consumer 1 first searches product A and only searches product $\mathrm{B}$ if the realized taste shock of product $\mathrm{A}$ is below product B's reservation utility. ${ }^{8}$ Hence, this

\footnotetext{
${ }^{7}$ The reservation utility is time invariant because it only depends on the search costs $c_{i j}$, product intercepts $\xi_{i j}$, and the time-invariant distribution of the taste shock $\varepsilon_{i j t}$.

${ }^{8}$ Because match values $\varepsilon_{i j t}$ have identical distribution across products, the ranking of reservation utilities in this model coincides with the ranking of product intercepts $\xi_{i j}$.
} 


\begin{tabular}{lccccc} 
Panel A: & \multicolumn{3}{c}{ True } & \multicolumn{3}{c}{ Purchase } \\
No Search Costs & \multicolumn{2}{c}{ Trobability } & Persistence \\
& Intercepts & A & B & \\
Product & A & B & 0.76 & 0.24 & 0.64 \\
\hline Consumer 1 & 0 & -1 & 0.24 & 0.76 & 0.64 \\
Consumer 2 & 0 & 1 & &
\end{tabular}

\begin{tabular}{lcccccccccc} 
Panel B: & \multicolumn{1}{c}{ Pearch Costs $=\mathbf{0 . 5}$} & \multicolumn{2}{c}{ True } & \multicolumn{2}{c}{ Search } & \multicolumn{2}{c}{ Purchase } & & \multicolumn{2}{c}{ Inferred } \\
& Intercepts & \multicolumn{2}{c}{ Probability } & \multicolumn{2}{c}{ Probability } & Persistence & Intercepts \\
Product & A & B & A & B & A & B & & A & B \\
\hline Consumer 1 & 0 & -1 & 1 & 0.12 & 0.91 & 0.09 & 0.84 & 0 & -1.93 \\
Consumer 2 & 0 & 1 & 0.12 & 1 & 0.09 & 0.91 & 0.84 & 0 & 1.93 \\
\hline
\end{tabular}

\begin{tabular}{|c|c|c|c|c|c|c|c|c|c|}
\hline \multirow{2}{*}{$\begin{array}{l}\text { Search Costs }=1 \\
\text { Product }\end{array}$} & \multicolumn{2}{|c|}{$\begin{array}{c}\text { True } \\
\text { Intercepts }\end{array}$} & \multicolumn{2}{|c|}{$\begin{array}{c}\text { Search } \\
\text { Probability }\end{array}$} & \multicolumn{2}{|c|}{$\begin{array}{c}\text { Purchase } \\
\text { Probability }\end{array}$} & \multirow[t]{2}{*}{ Persistence } & \multicolumn{2}{|c|}{$\begin{array}{c}\text { Inferred } \\
\text { Intercepts }\end{array}$} \\
\hline & $\mathrm{A}$ & B & $\mathrm{A}$ & B & A & B & & $\mathrm{A}$ & B \\
\hline Consumer 1 & 0 & -1 & 1 & 0.03 & 0.97 & 0.03 & 0.94 & 0 & -2.77 \\
\hline Consumer 2 & 0 & 1 & 0.03 & 1 & 0.03 & 0.97 & 0.94 & 0 & 2.77 \\
\hline
\end{tabular}

Table 1: The Impact of Search Costs on Purchase Shares: A Simple Example.

consumer only searches product B with probability

$$
\operatorname{SearchProb}_{1 B}=\operatorname{Pr}\left(0+\varepsilon_{i A t}<z_{1 B}\right)=0.12,
$$

where $z_{1 B}$ is the reservation utility from equation (2). ${ }^{9}$ If the consumer searches product $\mathrm{B}$, she then decides which product to buy based on the realized utilities of the two products. By contrast, if she does not search product $\mathrm{B}$, she never learns the realization of the shock $\varepsilon_{i B t}$ and purchases product A. Combining these two observations, we obtain that the consumer purchases product A with probability

$$
\begin{aligned}
& \operatorname{PurchProb}_{1 A}(c=0.5)=\operatorname{Pr}\left(0+\varepsilon_{i A t} \geq z_{1 B}\right) \\
& +\operatorname{Pr}\left(0+\varepsilon_{i A t}<z_{1 B}\right) \cdot \operatorname{Pr}\left(0+\varepsilon_{i A t} \geq-1+\varepsilon_{i B t} \mid 0+\varepsilon_{i A t}<z_{1 B}\right)=0.91 \text {. }
\end{aligned}
$$

The first term captures the case in which the consumer only searches product A, whereas the second term captures the probability of purchasing product A after having searched both products. As before, we repeat this computation for all consumer-product pairs and report the results in Panel B of Table 1. In this scenario with costly search, each consumer purchases the highest-intercept

\footnotetext{
${ }^{9}$ Reservation utilities do not have closed-form solutions for most distributions $F_{\varepsilon}(\varepsilon)$. Using a numerical approximation, we compute $z_{1 B}=-1.19$. Under the normality assumption, the probability of searching product $\mathrm{B}$ is then equal to $\Phi(-1.19)=0.12$ where $\Phi$ denotes the standard normal CDF.
} 
product with probability $91 \%$ and purchases the other product only with probability $9 \%$.

Comparing purchase probabilities under the two scenarios reveals that search costs tend to concentrate purchase probabilities around products with higher intercepts. This effect becomes even stronger with larger search costs, which we illustrate in Panel $\mathrm{C}$ of Table 1 where we increase search costs to $c=1.0$. Intuitively, higher search costs make consumers less likely to search the products with lower intercepts, thus reducing the purchase probabilities for these products. This shift in purchase probabilities towards high-intercept products also increases choice persistence, i.e., the probability that the consumer chooses the same product in both time periods. While this probability equals 0.64 in the perfect-information scenario, it increases to 0.84 when the search cost equals $c=0.5$ and further increases to 0.94 when the search cost equals $c=1.0$.

With costly search, consumers make more extreme choices. To see this, consider how consumers 1 and 2 change their probability of buying product $A$ as we increase search costs. In the perfectinformation scenario, the purchase probabilities of both consumers equal 0.76 and 0.24 . However, these probabilities become 0.91 and 0.09 when search costs equal 0.5 and further increase to 0.97 and 0.03 when search costs increase to 1.0. Hence, costly search increases the dispersion of purchase probabilities for product A across consumers. By symmetry, the same happens to the dispersion of purchase probabilities for product B.

This heterogeneity in purchase probabilities is driven by both preference heterogeneity and search costs. Ignoring search costs forces us to fully attribute heterogeneity in choices to preferences, thus generating an upward bias in the estimates of preference heterogeneity. To illustrate, we use purchase probabilities from Table 1 to infer product intercepts of the two consumers assuming perfect information. For example, when the purchase probability of consumer 1 for product B equals 0.91 , we infer the intercept $\hat{\xi}_{1 B}$ by solving the equation $\operatorname{Pr}\left(0+\varepsilon_{i A t}>\hat{\xi}_{1 B}+\varepsilon_{i B t}\right)=0.91 .^{10}$ We compute the intercept of consumer $2, \hat{\xi}_{2 B}$, in a similar fashion. According to the results, the inferred intercepts $\hat{\xi}_{1 B}$ and $\hat{\xi}_{2 B}$ are larger in absolute terms than the true product intercepts. For example, when search costs are 0.5 , we infer the intercepts to be -1.93 and 1.93 which are more dispersed than the true values -1 and 1 . Raising search costs to 1 further exacerbates this bias, generating the inferred intercepts -2.77 and 2.77. As a whole, these results show that ignoring search costs leads to an overestimation of preference heterogeneity, and that the magnitude of the bias is proportional to consumers' search costs.

\section{$3.2 \quad$ Product-Specific Search Costs}

So far we assumed identical search costs across products. One might think, however, that search costs may vary across products. For example, an online retailer may make some products more salient than others (e.g, via advertising or product rankings), thus lowering these products' search costs. To illustrate the implications of product-specific search costs, we repeat the exercise from the previous section but assume that search costs are asymmetric across products. In particular, we assume a search cost of 0.5 for product A and 2.0 for product B. Given those search cost values,

\footnotetext{
${ }^{10}$ For this exercise, we treat the consumer-specific purchase probabilities as observable.
} 


\begin{tabular}{lcccc} 
Panel A: & \multicolumn{2}{c}{ True } & \multicolumn{2}{c}{ Purchase } \\
No Search Costs & Intercepts & \multicolumn{2}{c}{ Probability } \\
Product & A & B & A & B \\
\hline Consumer 1 & 0 & -1 & 0.76 & 0.24 \\
Consumer 2 & 0 & 1 & 0.24 & 0.76 \\
\hline
\end{tabular}

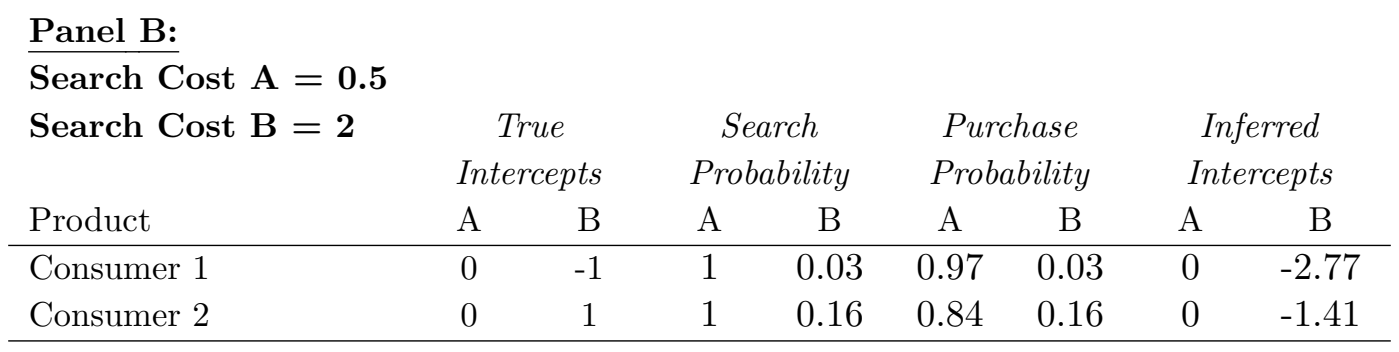

Table 2: Purchase Behavior when Search Costs are Asymmetric Across Products.

both consumers find it attractive to search product A first. Therefore, the search order of consumer 2 no longer aligns with her preferences and is driven by the difference in product-specific search costs. As illustrated in Table 2, both consumers start by searching product A and are more likely to buy it than product B. As a result, when costly search is ignored, we mistakenly conclude that these consumers' preferences are similar, which leads to an underestimation of preference heterogeneity.

As this specific example shows, introducing asymmetric search costs may reverse the conclusions from section 3.1 and may lead to an underestimation (rather than overestimation) of preference heterogeneity. We therefore explicitly model product-specific search costs in our application (see section 4). However, even when allowing for possible asymmetries in search costs, we nevertheless find that preference heterogeneity is significantly overestimated when ignoring search frictions.

\subsection{Many Products and Continuous Distribution of Heterogeneity}

The examples we discussed thus far are somewhat stylized in that they feature only two consumers and two products. We now present a more general example whose structure is more closely related to the empirical model we use for estimation. This example mimics the preference structure of random-coefficient demand models which have been extensively used in the prior literature to estimate preference heterogeneity. We use the same utility specification as before (see equation 1) but consider a market with three products and assume that the consumer-specific intercepts for each of these products follow a standard normal distribution. We simulate search behavior for a large number of consumers and a large number of search sessions for each consumer. ${ }^{11}$

In the left graph in Figure 1, we plot the distribution of resulting purchase probabilities across

\footnotetext{
${ }^{11}$ Note that we simplify this example by assuming identical search costs across products. One should keep in mind, however, that introducing differences in product-specific search costs may potentially tilt results towards underestimating preference heterogeneity (see the discussion in section 3.2).
} 

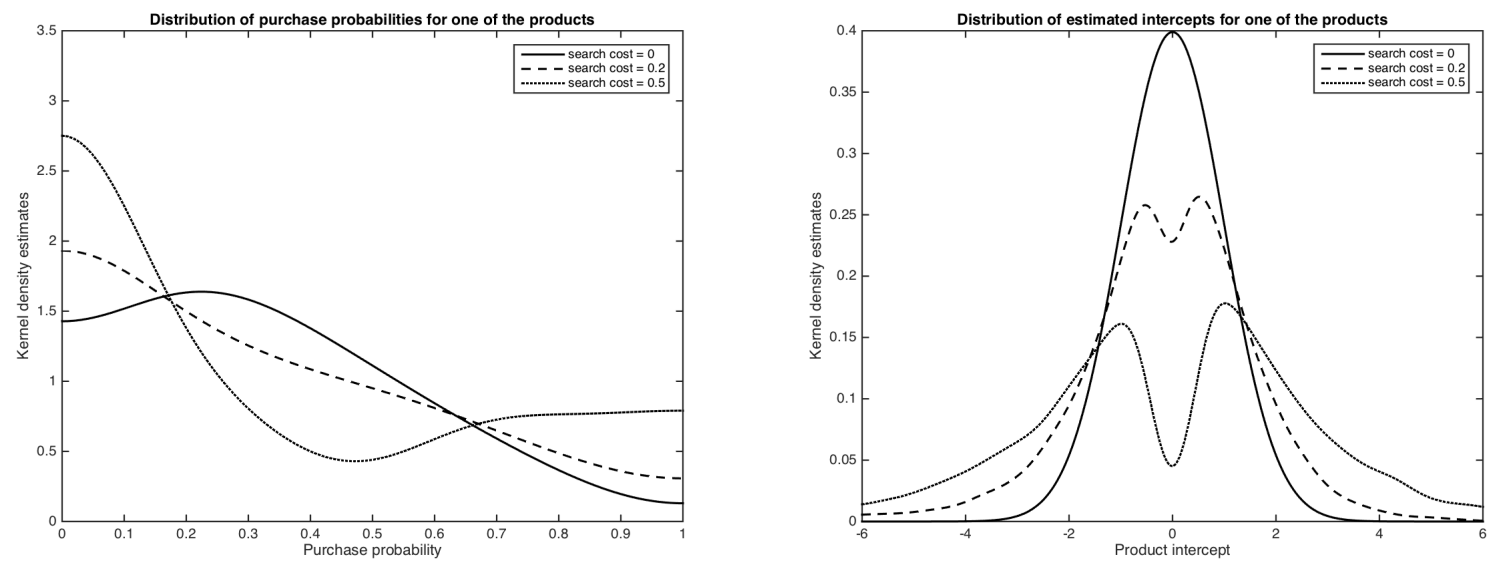

Figure 1: Distribution of Purchase Probabilities and Estimated Product Intercepts for Different Values of Search Costs.

consumers for one of the products. ${ }^{12}$ The solid line shows the distribution of purchase probabilities in the perfect-information case. Increasing search costs from 0 to 0.2 shifts the probability mass toward the extremes by increasing the mass of consumers with low and high purchase probabilities and decreasing the mass of consumers with intermediate purchase probabilities (dashed line). As we further increase search costs, this pattern becomes more pronounced (dotted line). Similar to our previous example, the increase in search costs also increases choice persistence. The average choice persistence increases from 0.44 in the perfect-information case to 0.65 in the case where search costs are 0.5 .

Next, we infer product intercepts assuming perfect information. The right-hand graph in Figure 1 shows the density of inferred intercepts for one of the products under different search costs. The solid line depicts the benchmark case with zero search costs; in this case, the distribution of inferred intercepts coincides with the true distribution. As search costs increase, the density of estimated intercepts shifts from the middle toward the extremes. Thus, similar to our previous example with two consumers and two products, ignoring search costs leads to an overestimation of preference heterogeneity.

We also consider a similar example for the case of a market that is characterized by strong vertical differentiation of products. To introduce vertical differentiation, we assume that the intercepts follow a normal distribution with an identical standard deviation of 0.2 and with means 0,1 , and 2 . That is, most consumers now agree which products look ex-ante more attractive, but they slightly differ in the exact values of their product-specific intercepts. We report the distributions of inferred intercepts for products 2 and 3 in Figure 2. For both products, the variance of inferred intercepts increases when search costs are higher. Moreover, the distributions are shifted to the right under costly search relative to the perfect-information case. Hence, in the vertical case, ignoring search

\footnotetext{
${ }^{12}$ Because the intercepts follow identical distributions for these three products, the distributions of resulting purchase probabilities are also identical.
} 

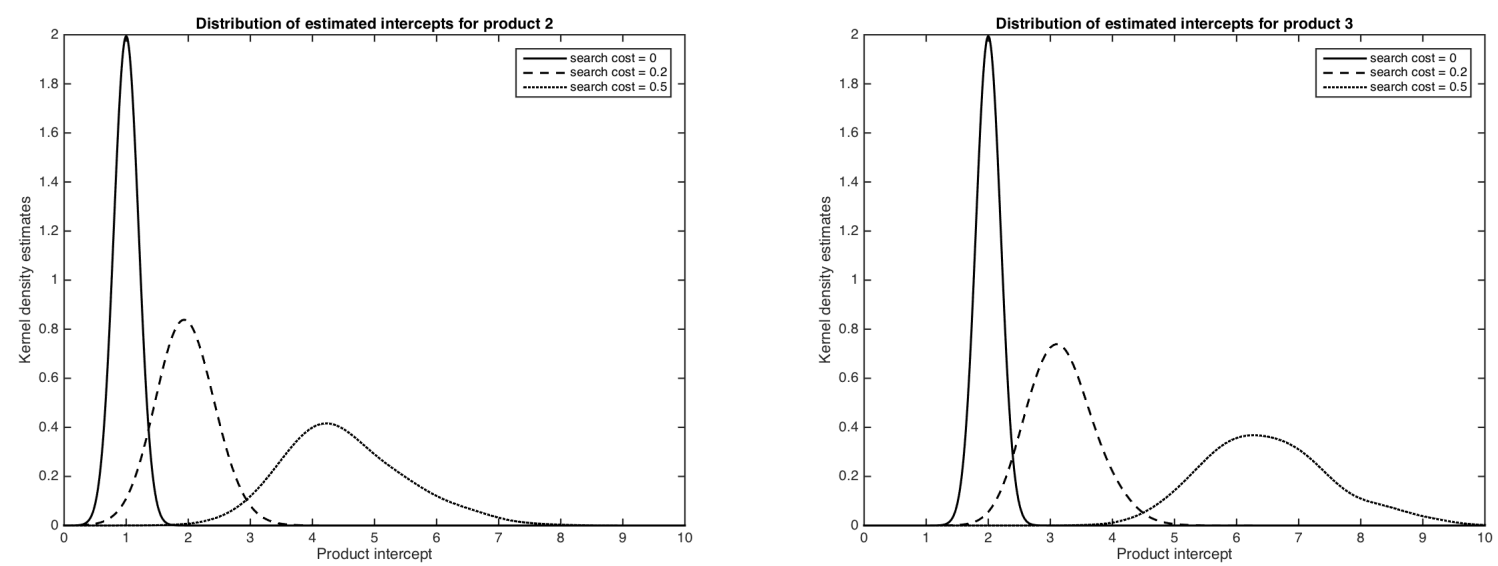

Figure 2: Vertical Differentiation Case. The two graphs display kernel density estimates for the distribution of inferred product intercepts for product 2 (left) and product 3 (right).

leads to a bias in both the variance of inferred intercepts as well as mean intercepts. While our focus is on the estimates of preference heterogeneity, i.e. the variance of intercepts, we later also document a bias in inferred mean intercepts in our empirical application. Average persistence in choices increases from 0.51 in the perfect-information case to 0.78 in the case when search costs are equal to 0.5 .

\subsection{General Case: Search Costs Increase Persistence and Inferred Preference Heterogeneity}

The examples above illustrate that when search costs increase, consumer choices tend to become more persistent. As a result, consumers appear to have strong preferences for certain items, which may lead to an overestimation of preference heterogeneity when the researcher ignores costly search. In this section and in Appendices A and B, we show that higher search costs lead to higher choice persistence and inferred preference heterogeneity under a wide range of assumptions about model primitives. We emphasize that this relationship between search costs, choice persistence, and inferred preference heterogeneity only applies to a setting where search costs are common across products. As the example outlined in Section 3.2 shows, our findings do not apply to product-specific changes in search costs; therefore, it is an empirical question whether search cost asymmetries are large enough such that preference heterogeneity is underestimated. In our application we find that even when allowing for product-specific search costs, preference heterogeneity is significantly overestimated.

Suppose a consumer chooses from $J$ products indexed by $j$, and that the indirect utility is $u_{j}=\xi_{j}+\varepsilon_{j}$ where $\xi_{j}$ is product $j$ 's intercept observed prior to search and $\varepsilon_{j}$ is the taste shock revealed during search. Assume $\varepsilon_{j}$ is independently and identically distributed across products and sessions according to some continuous distribution $F(\varepsilon)$. In this setting, we establish two results.

1. In the sequential search model with uniform search costs across products, increasing search 
costs by $\Delta>0$ leads to an increase in choice persistence for any value of $\Delta$.

2. In the sequential search model with uniform search costs across products, increasing search costs by $\Delta>0$ leads to an increase in the inferred heterogeneity of product intercepts for any value of $\Delta$.

Intuitively, because search is directed, the consumer always starts by searching products with high intercepts. As search costs increase, the consumer increasingly focuses on searching a small set of products with the largest intercepts, gradually converging to the state where she only searches and purchases the product with the largest intercept $\xi_{j}$. As a result, the consumer's choices become increasingly concentrated around a few products, which increases the probability of purchasing the same product in consecutive search sessions. In Appendix A, we provide a formal proof of this result which holds for all continuous distributions of taste shocks $F(\varepsilon)$. The result also applies to markets where consumers search over prices, in which case we can interpret taste shocks $\varepsilon_{j}$ as consumer's preference over prices and $F(\varepsilon)$ as a price distribution consistent with consumer's beliefs. We also show in the same appendix that this result holds for the simultaneous search model of Chade and Smith (2006).

Furthermore, when search costs are positive, the bias in the inferred intercept of a given product, $\left(\hat{\xi}_{j}-\xi_{j}\right)$, increases in the true intercept's value $\xi_{j}$. It therefore follows that for any pair of consumers (denoted as consumer 1 and consumer 2) with $\xi_{1 j}>\xi_{2 j}$ it holds that inferred intercepts are further apart from each other than true intercepts, so that $\hat{\xi}_{1 j}-\hat{\xi}_{2 j}>\xi_{1 j}-\xi_{2 j}$. Therefore, search costs increase the dispersion in inferred intercepts across consumers. Furthermore, the bias in inferred intercepts is negative (positive) if the true intercept value is negative (positive). Therefore, mean intercepts are biased away from zero. Finally, the absolute value of the bias increases in search costs, thus leading to a stronger increase in dispersion if search costs are higher. In Appendix B we use an extensive set of simulations to show that this result holds for the utility specification outlined above and under various assumptions regarding the distribution of taste shocks $\varepsilon_{j}{ }^{13}$

\section{Empirical Model and Identification}

To further illustrate that ignoring search costs leads to biased heterogeneity estimates, we now consider an empirical example. Our general strategy in the empirical application is to use a dataset most suitable for recovering a flexible distribution of preference heterogeneity. To this end, we use a unique panel dataset in where each consumer visits the retailer's website multiple times, and in which we observe search and purchase behavior during each consumer visit.

To estimate model's parameters from such data, we first extend the sequential search model from section 2, making it compatible with panel data. The extended model adds price to the utility function and includes an additional taste shock revealed prior to search. We then derive restrictions

\footnotetext{
${ }^{13}$ Although we were unable to formally prove this result, we demonstrate it using simulations. To this end, we simulate consumer behavior and compute inferred product intercepts under a variety of different modeling assumptions.
} 
that observed choices impose on model parameters and realizations of taste shocks and finish by discuss the identification of model parameters.

\subsection{Panel Data Framework}

As before, suppose each consumer $i$ conducts $T_{i}$ search sessions indexed by $t=1, \ldots, T_{i}$. In each session, she chooses exactly one product out of $J$ available alternatives. The utility consumer $i$ derives from purchasing product $j$ in search session $t$ equals

$$
u_{i j t}=\delta_{i j t}+\varepsilon_{i j t}=\left(\xi_{i j}-\alpha_{i} p_{i j t}+\mu_{i j t}\right)+\varepsilon_{i j t} .
$$

In this expression, $\xi_{i j}$ is a time-invariant intercept capturing consumer $i$ 's preferences for product $j ; p_{i j t}$ denotes the price of product $j$ in session $t$ of consumer $i{ }^{14} \alpha_{i}$ denotes the price coefficient; and $\mu_{i j t}$ and $\varepsilon_{i j t}$ are idiosyncratic taste shocks, both distributed normally and i.i.d. with zero mean and variances $\sigma_{\epsilon}^{2}$ and $\sigma_{\mu}^{2}$. Prior to searching, a consumer knows the realizations of $\delta_{i j t}$ and the distribution of $\varepsilon_{i j t}$ but has to search in order to learn the realization of $\varepsilon_{i j t}{ }^{15}$ The consumer pays a fixed cost $c_{i j}$ when she searches product $j$. We assume that search costs may differ across products, reflecting that some products might be ranked higher on the webpage and hence are more salient to consumers (Ursu, 2018). We model these differences by assuming that the search cost $c_{i j}$ is drawn from a log-normal distribution with product-specific location parameters. As before, the model does not include an outside option.

The key difference relative to the framework in section 2 is the pre-search taste shock $\mu_{i j t}$. Absent this taste shock, an individual consumer who encounters the same product prices in each search session would always search products in the same order. Introducing the shock $\mu_{i j t}$ resolves this issue by adding a source of randomness in pre-search utilities. One can interpret the taste shock $\mu_{i j t}$ as an unobserved information shock that changes the consumer's propensity to search different products. Such a shock may include recommendations from friends or other product information acquired before the start of the search session. By contrast, the post-search taste shock $\varepsilon_{i j t}$ represents any information retrieved from the product page itself such as information from customer reviews and detailed product descriptions.

In our main specification, we assume shocks $\mu_{i j t}$ and $\varepsilon_{i j t}$ are independent across search sessions. This assumption implies that new information is available in each search session. We later relax this assumption in section 6.4 where we re-estimate the model allowing for information to be correlated across search sessions. We find that the estimates of preference heterogeneity change little compared to the ones from our main specification.

We normalize the variances of taste shocks at $\sigma_{\mu}^{2}=1$ and $\sigma_{\varepsilon}^{2}=1$. The first normalization is necessary to fix the scale of utility. We could in principle relax the second one and estimate

\footnotetext{
${ }^{14}$ Consumers who purchase on the same day face identical prices. Price is indexed with an $i$ subscript because $t$ denotes a search session rather than calendar time.

${ }^{15}$ This assumption implies consumers observe prices before searching. The assumption is natural in our application, because users of the online store start the session by browsing a list of products that shows product names, photos, and prices.
} 
$\sigma_{\varepsilon}^{2}$ from the data, but it is generally hard to estimate this variance separately from search costs. Increasing the variance $\sigma_{\varepsilon}^{2}$ leads to an increase in the benefits from search, which makes consumers search more, thus producing an effect similar to that of reducing search costs. We address this issue by normalizing the variance $\sigma_{\varepsilon}^{2}$. Experimenting with different values of $\sigma_{\varepsilon}^{2}$ reveals that this second normalization has little impact on our qualitative results. In particular, when we change the normalized standard deviation $\sigma_{\varepsilon}$ by a factor of $k$, estimated search costs change roughly by a factor $1 / k$. Because search costs and the variance of post-search shocks are not fully collinear, other parameter estimates also change albeit only marginally. Most importantly, in our application we find that the monetized values of preference heterogeneity estimates, the primary object of interest in this paper, remain almost unchanged when altering the value of $\sigma_{\varepsilon}^{2} \cdot{ }^{16} \mathrm{In}$ Appendix $\mathrm{C}$, we discuss in more detail why these two variance normalizations are necessary for the estimation.

\subsection{Restrictions Imposed by Observed Choices}

The search model above imposes a set of restrictions on the model's parameters and utility shocks. In this section we derive three sets of restrictions associated with different decisions: in which order to search products, when to stop searching, and what to purchase.

\section{Search order}

According to the optimal search rule, the consumer searches products in order of decreasing reservation utilities (see section 2). Let $\Im=\{1, \ldots, J\}$ denote the set of available products, and let $S_{i t} \subseteq \Im$ be the set of products that consumer $i$ searched during search session $t$. Also let $M_{i t}$ be the number of products in this set so that $\left|S_{i t}\right|=M_{i t}$. Without loss of generality, throughout this section we index products in the order in which they were searched by the consumer in a given search session, i.e. product $j=1$ is searched first, $j=2$ searched second, and so on.

When the consumer searches products $S_{i t}$, the ranking of reservation utilities $z_{i j t}$ must be consistent with the observed search order:

$$
z_{1 i t} \geq z_{2 i t} \geq \cdots \geq z_{M_{i t}, i t} \geq z_{k i t} \text { for } \forall k \notin S_{i t}
$$

Because we only observe search order for products that were actually searched, the data does not impose any restrictions on the ranking of reservation utilities for unsearched products. We only know that the reservation utility of the product searched last, $z_{M_{i t}, i t}$, exceeds the reservation utilities of all unsearched products. A more compact way to write the inequalities in (5) is

$$
z_{m i t} \geq \max _{l \in S_{m i t}} z_{l i t} \text { for } m=1, \ldots, M_{i t},
$$

\footnotetext{
${ }^{16}$ Consumers' stopping decisions depend on the ratio of search costs and $\sigma_{\epsilon}^{2}$, whereas purchase decisions only on $\sigma_{\epsilon}^{2}$. Therefore, $\sigma_{\epsilon}^{2}$ could be in principle be identified by the degree of randomness in purchase decisions (a larger value of $\sigma_{\epsilon}^{2}$ would lead to higher randomness in purchase decisions). In practice we found that $\sigma_{\epsilon}^{2}$ is hard to estimate and hence we normalize it.
} 
where $S_{m i t}=\Im \backslash\{j: j \leq m\}$ represents the set of products left unsearched after searching options $1, \ldots, m$. These inequalities specify the restrictions that the observed order of search imposes on parameters and realizations of taste shocks.

\section{Continuation and stopping decisions}

The consumer continues searching if and only if the maximum realized utility among searched products is lower than the maximum reservation utility among unsearched options. If the consumer decides to search product $m$, it must be that the maximum realized utility among searched products $1, \ldots,(m-1)$ is lower than the reservation utility of product $m$, namely, $z_{m i t}$. This relationship must hold for all searched products except the first one, because in our model the consumer always searches at least one product:

$$
\max \left\{u_{1 i t}, \ldots, u_{(m-1) i t}\right\} \leq z_{m i t} \text { for } m=2, \ldots, M_{i t} .
$$

If the consumer decides to stop after searching product $M_{i t}$, the maximum realized utility of searched products must exceed the reservation utilities of all unsearched products: ${ }^{17}$

$$
\max \left\{u_{1 i t}, \ldots, u_{M_{i t}, i t}\right\} \geq \max _{k \notin S_{i t}} z_{k i t}
$$

The inequalities in (6) and (7) capture the restrictions the observed continuation and stopping decisions impose on the parameters and realizations of taste shocks.

\section{Purchase decision}

Upon finishing the search, the consumer purchases the product with the highest realized utility among the searched options. Therefore, if the consumer searches products $S_{i t}$ and buys a product $y_{i t} \in S_{i t}$, this product's realized utility should be the largest among all searched products:

$$
u_{y_{i t}, i t} \geq \max _{j \in S_{i t}} u_{j i t}
$$

\subsection{Identification}

We build our identification argument around the panel dimension of the dataset. In particular, we discuss how individual-level preference parameters $\left(\xi_{i 1}, \ldots, \xi_{i J}, \alpha_{i}\right)$ and search $\operatorname{costs}\left(c_{i 1}, \ldots, c_{i J}\right)$ can be identified for one individual consumer when the researcher observes the probability distribution of search and purchase decisions for that consumer. Given that these individual-level parameters are identified, it then follows that the distribution of these parameters across individuals is nonparametrically identified. Because our argument requires us to observe the probability distribution of searches and purchases for each consumer, the argument effectively assumes that the data includes

\footnotetext{
${ }^{17}$ The consumer will also stop searching if she has exhausted all search opportunities. In this case, the stopping inequality is irrelevant.
} 
an infinitely large number of search sessions per consumer. Note, however, that the data in our empirical application only contains a moderate panel dimension. Therefore, instead of estimating individual-level parameters for each consumer, in our application we estimate the parameters of the distribution from which preferences and search costs are drawn.

To the best of our knowledge, when only cross-sectional data is available, the prior literature provides no formal identification argument for the nonparametric identification of consumer heterogeneity. Several papers argue that, when only cross-sectional data is available, the researcher needs to rely on functional form assumptions to identify unobserved preference heterogeneity. For example, Honka (2014) argues that preference heterogeneity can be identified through deviations of demand patterns from the independence of irrelevant alternatives (IIA) property, which follows from the assumption that error terms follow the extreme value distribution in her model. Honka and Chintagunta (2017) and Yavorsky, Honka, and Chen (2020) only allow for observed heterogeneity and argue that unobserved heterogeneity in preference is generally not identified with cross-sectional data. ${ }^{18}$ Unlike these papers that do not explicitly focus on estimating unobserved heterogeneity, the primary goal of our paper is to study how estimates of preference heterogeneity are affected by search frictions. Therefore, it is particularly important in our setting to achieve nonparametric identification through the use of panel data.

\section{Inferring Reservation Utilities}

To simplify exposition, we first assume that price does not enter the utility function and focus on the identification of product intercepts and search costs. During search session $t$, consumer $i$ searches products in order of decreasing reservation utilities $z_{i j t}$ as defined in equation (2). Following Kim, Albuquerque, and Bronnenberg (2010), we decompose the reservation utility as $z_{i j t}=\bar{z}_{i j}+\mu_{i j t}=$ $\xi_{i j}+g\left(c_{i j}\right)+\mu_{i j t}$ where $g\left(c_{i j}\right)$ is a known function that monotonically decreases in the search cost and depends only on the search cost $c_{i j}$ and the distribution of preference shocks $\varepsilon_{i j t} .{ }^{19}$ Going forward, we refer to the deterministic part of the reservation utility $\bar{z}_{i j}$ as mean reservation utility. The probability that consumer $i$ first searches product $k$ can be expressed as:

$$
\operatorname{Pr}(i \text { searches product } k \text { first })=\operatorname{Pr}\left(z_{k i t} \geq z_{j i t} \quad \forall j\right)=\operatorname{Pr}\left(\bar{z}_{i k}+\mu_{i k t} \geq \bar{z}_{i j}+\mu_{i j t} \quad \forall j\right) \text {. }
$$

Because we assume preference shocks $\mu_{i j t}$ follow the standard normal distribution, the expression in (9) corresponds to the standard probit probability. However, unlike in standard discrete choice models where probit expressions describe purchase probabilities, here these expressions capture the probabilities that different products are searched first within a search session. ${ }^{20}$ In a setting with panel data, we can observe first-search probabilities for each consumer $i$ and

\footnotetext{
${ }^{18}$ Yavorsky, Honka, and Chen (2020) state that "To identify unobserved heterogeneity in a continuous mixture model, a researcher must observe consumers more than once, i.e., the data must have a panel structure."

${ }^{19} \mathrm{Kim}$, Albuquerque, and Bronnenberg (2010) derive the exact shape of the reservation utility in the appendix of their paper.

${ }^{20}$ In this sense, data on first searches in our setting provides information similar to panel data on purchases in perfect-information models.
} 
each product $j$. We emphasize that without panel data, we would only observe one search session per consumer; in that case, we would not be able to compute these consumer-specific probabilities. Following Hotz and Miller (1993), we invert the observed first-search probabilities $\operatorname{Pr}(i$ searches product 1 first $), \ldots, \operatorname{Pr}(i$ searches product $J$ first $)$ to infer the values of $\bar{z}_{i j}$. Hence, for each consumer $i$, we infer the $\bar{z}_{i j}$ term for all products.

The identities of other searched products provide additional information about model parameters. Based on the inequalities in (5), consumer $i$ chooses to search products in a specific order with probability

$$
\operatorname{Pr}\left(i \text { searches in order } 1, \ldots, M_{i t}\right)=\operatorname{Pr}\left(z_{m i t} \geq \max _{l \in S_{m i t}} z_{\text {lit }} \text { for } m=1, \ldots, M_{i t}\right)
$$

This expression suggests that products that are often searched early during the search process should have higher mean reservation utilities $\bar{z}_{i j}$. Similarly, the mean reservation utility should be low for products that are searched at the end of the search session or not searched at all. This additional information about the complete search order, not just about first searches, helps us estimate preference parameters.

\section{Separating Preferences from Search Costs}

Next, we decompose the mean reservations utilities into preferences and search costs. The probability that consumer $i$ in session $t$ stops after $M_{i t}$ searches is given by:

$$
\operatorname{Pr}\left(i \text { stops after searching } M_{i t} \text { products }\right)=\operatorname{Pr}\left(\max \left\{u_{1 i t}, \ldots, u_{M_{i t}, i t}\right\} \geq \max _{k \notin S_{i t}} z_{k i t}\right)
$$

Note that the stopping decision depends on the realized utilities of products that were searched and reservation utilities of products that have not yet been searched. Intuitively, search costs for products that have already been searched are not relevant with regards to the stopping decision, but only realized utilities of searched products matter. The irrelevance of search costs of already searched products allows us to separately identify preferences and search costs. In particular, suppose we increase search costs for one specific product $c_{i j}$ but at the same time increase this product's intercept $\xi_{i j}$ such that $\bar{z}_{i j}$ remains unchanged. ${ }^{21}$ Since $\bar{z}_{i j}$ is unchanged, the expected search order remains unchanged as well. However, the realized utility of product $j$ is now higher due to its higher intercept; therefore, according to equation (11), the consumer is more likely to stop searching after having searched this specific product. Hence, conditional on $\bar{z}_{i j}$, stopping decisions of consumers conditional on searching a specific product allows us to identify the product's intercept. Finally, having identified $\bar{z}_{i j}$ and $\xi_{i j}$, we can find the value of $c_{i j}$ such that the relationship

\footnotetext{
${ }^{21}$ The function $g\left(c_{i j}\right)$ which enters the reservation utility $z_{i j t}$ is monotonically decreasing in search costs and ranges from minus infinity to plus infinity as we vary search costs from infinity to zero (Kim, Albuquerque, and Bronnenberg, 2010). Therefore, we can always change the search cost and the product intercept so that the mean reservation utility $\bar{z}_{i j}$ remains unchanged.
} 
$\bar{z}_{i j}=\xi_{i j}+g\left(c_{i j}\right)$ holds. This logic can be applied repeatedly for different $i$ and $j$, implying that we can identify product intercepts and product-specific search costs for all consumers and products.

We have so far ignored the price coefficient and discussed identification of product intercepts; however, identification of the price coefficient is straightforward. The extent to which a consumer searches cheaper products earlier in a search session identifies her price coefficient $\alpha_{i}$ as long as price variation is exogenous with respect to taste shocks. We maintain this exogeneity assumption in our empirical application. Because the model includes time-invariant product intercepts, identification of the price coefficient comes from price changes for the same product over time. Hence, our main identifying assumption is that the timing of price changes is uncorrelated with temporary preference shocks.

In summary, our identification argument establishes that with panel data, we can identify the product intercepts $\xi_{i j}$, price coefficient $\alpha_{i}$, and search $\operatorname{costs} c_{i j}$ for each individual consumer. Since this logic can be applied to each consumer, we are able to nonparametrically identify the distribution of preferences and search costs across consumers.

\section{The Role of Purchase Data}

The arguments presented thus far suggest that search order and stopping decisions are sufficient to identify preferences and search costs for a given consumer. We note, however, that consumers' purchases conditional on search decisions provide additional variation to estimate preferences separately from search costs. The corresponding argument is somewhat involved, so we relegate a more detailed discussion to Appendix D. The key pattern we outline there is that increasing a product's intercept makes it more likely that this product will be purchased conditional on being searched. This observation holds as long as the product in question was not searched last within the search session. In other words, increasing the intercept increases the product's recall probability, defined as the probability of purchasing a product searched earlier in the search sequence. At the same time, changing a product's search cost does not change the product's recall probability. Therefore, product-specific recall rates provide additional information to recover preferences separately from search costs.

\section{Estimation}

\subsection{Parametrization}

Our identification argument above assumes that the data includes an infinite number of search sessions per consumer. In our actual dataset, however, panel dimension is much longer for some consumers than others, as the number of sessions per consumer ranges from 2 to 35 . To handle data for consumers with a limited panel dimension, we impose additional assumptions on the distribution of consumer heterogeneity that allow us to pool information across consumers. Therefore, unlike in the nonparametric argument above where panel data identifies consumer-specific search 
cost and preference parameters, in our empirical model below panel data helps us estimate the hyperparameters governing the distribution of consumer heterogeneity.

First, we assume that product intercepts $\xi_{i j}$ for each product $j$ follow a normal distribution:

$$
\xi_{i j} \sim N\left(\bar{\xi}_{j}, \sigma_{\xi_{j}}^{2}\right)
$$

where $\bar{\xi}_{j}$ is the mean intercept for product $j$, and $\sigma_{\xi_{j}}^{2}$ denotes the variance of the product intercept that captures unobserved preference heterogeneity. Second, we assume that price coefficients $\alpha_{i}$ also vary across consumers, capturing the differences in the marginal utility of income. To ensure price coefficients are positive for all consumers, we assume that $\alpha_{i}$ follows a log-normal distribution such that

$$
\log \alpha_{i} \sim N\left(\bar{\alpha}, \sigma_{\alpha}^{2}\right)
$$

where $\bar{\alpha}$ and $\sigma_{\alpha}^{2}$ denote the mean and the variance. Similarly, we assume that the search cost $c_{i j}$ for product $j$ follows a log-normal distribution with a product-specific mean $\overline{c_{j}}$ and common variance $\sigma_{c}^{2}$ :

$$
\log c_{i j} \sim N\left(\overline{c_{j}}, \sigma_{c}^{2}\right)
$$

We do not model observed heterogeneity for several reasons. In our empirical application, we do not have access to any demographic variables that might be used as predictors for consumers' preferences and search costs. This situation is relatively common in online markets where firms tend to have rich information on consumers' purchase histories but relatively little information on their demographics. Furthermore, many studies in the literature on preference heterogeneity show that demographic variables explain only a small share of preference heterogeneity relative to the unobserved component (Rossi and Allenby, 1993; Rossi, McCulloch, and Allenby, 1996; Allenby and Rossi, 1999). Hence, modeling observed heterogeneity may not be crucial for explaining the observed behavior of consumers.

We also note that in several papers, search costs have been parameterized as a function of the time at which the search occurs (Koulayev, 2014; Chen and Yao, 2017) or as a function of the product's ranking on the website (Ursu, 2018). Unfortunately we do not have access to any characteristics of this kind and hence we do not model search costs as a function of observable consumer characteristics.

\section{$5.2 \quad$ Likelihood Function}

To derive the likelihood function, we first summarize the restrictions that observed choices impose on parameters and taste shocks. Let vector $\theta_{i}=\left(\xi_{i 1}, \ldots, \xi_{i J}, \alpha_{i}, c_{i 1}, \ldots, c_{i J}\right)$ denote the consumer's type that describes her product intercepts, price sensitivity, and product-specific search costs. Additionally, let $p_{i t}$ denote a $J \times 1$ price vector. If a consumer with type $\theta_{i}$ searches products $S_{i t}$ in order $\left\{1, \ldots, M_{i t}\right\}$ and purchases product $y_{i t} \in S_{i t}$, the following inequalities must hold: 


$$
\begin{gathered}
w_{m i t}^{O}\left(\theta_{i}, \mu_{i t}, \varepsilon_{i t}, p_{i t}\right)=z_{m i t}-\max _{l \in S_{m i t}} z_{l i t} \geq 0 \quad \text { for } \quad m=1, \ldots, M_{i t} \\
w_{m i t}^{S}\left(\theta_{i}, \mu_{i t}, \varepsilon_{i t}, p_{i t}\right)=z_{m i t}-\max \left\{u_{1 i t}, \ldots, u_{(m-1) i t}\right\} \geq 0 \quad \text { for } \quad m=2, \ldots, M_{i t} \\
w_{\left(M_{i t}+1\right) i t}^{S}\left(\theta_{i}, \mu_{i t}, \varepsilon_{i t}, p_{i t}\right)=\max \left\{u_{1 i t}, \ldots, u_{M_{i t}, i t}\right\}-\max _{k \notin S_{i t}} z_{k i t} \geq 0 \\
w_{i t}^{P}\left(\theta_{i}, \mu_{i t}, \varepsilon_{i t}, p_{i t}\right)=u_{y_{i t}, i t}-\max _{j \in S_{i t}} u_{j i t} \geq 0,
\end{gathered}
$$

where (15) corresponds to the order of search, (16) and (17) describe inequalities for continuation and stopping decisions, and (18) captures purchase inequalities. To simplify notation, we define function $W_{i t}\left(\theta_{i}, \mu_{i t}, \varepsilon_{i t}, p_{i t}\right)$ as

$$
W_{i t}\left(\theta_{i}, \mu_{i t}, \varepsilon_{i t}, p_{i t}\right)=\min \left\{w_{1 i t}^{O}, \ldots, w_{M_{i t}, i t}^{O}, w_{2 i t}^{S}, \ldots, w_{\left(M_{i t}+1\right) i t}^{S}, w_{i t}^{P}\right\} .
$$

so that $W_{i t}\left(\theta_{i}, \mu_{i t}, \varepsilon_{i t}, p_{i t}\right) \geq 0$ if and only if all the inequalities in (15)-(18) hold.

We now formulate the likelihood of the model. Suppose a researcher observes data on search and purchase decisions as well as prices of products at the time of each decision. Let $D_{i t}$ denote these data for consumer $i$ and session $t$, and let $D$ denote the data for all consumers and sessions. Suppose the data $D$ includes $N$ consumers, and each consumer $i$ conducts $T_{i}$ search sessions. The likelihood of observing search and purchase decisions of consumer $i$ in session $t$ given consumer's type $\theta_{i}$ and prices $p_{i t}$ is

$$
L_{i t}\left(D_{i t} \mid \theta_{i}, p_{i t}\right)=\operatorname{Pr}\left(W_{i t}\left(\theta_{i}, \mu_{i t}, \varepsilon_{i t}, p_{i t}\right) \geq 0 \mid \theta_{i}, p_{i t}\right)
$$

where the uncertainty on the right-hand side comes from taste shocks $\mu_{i t}$ and $\varepsilon_{i t}$, both of which are unobserved to a researcher.

Combining individual likelihoods, we can write the full likelihood of the data $D$ as

$$
L(D \mid \Omega, p)=\prod_{i=1}^{N} \int\left(\prod_{t=1}^{T_{i}} L_{i t}\left(D_{i t} \mid \theta_{i}, p_{i t}\right)\right) \cdot d F\left(\theta_{i} \mid \Omega\right),
$$

where $\Omega=\left(\bar{\alpha}, \sigma_{\alpha}, \bar{c}, \sigma_{c}, \bar{\xi}_{j}, \sigma_{\xi_{j}} \forall j\right)$ denotes the vector of hyperparameters that determine how types $\theta_{i}$ are distributed across consumers. Because types $\theta_{i}$ are unobserved to the researcher, the likelihood function in (21) contains an expectation of consumer-specific likelihoods with respect to the joint distribution of $\theta_{i}$. This joint distribution, $F\left(\theta_{i} \mid \Omega\right)$, is fully defined by the distributional assumptions in (12)-(14). 


\subsection{Importance Sampling}

A straightforward approach to estimation would be to maximize the simulated version of the likelihood (21) with respect to parameters $\Omega$. In our application, this approach is impractical because we need to recompute the simulated likelihood for each new guess of parameters. This repeated computation is computationally burdensome because we need to recompute reservation utilities and simulate consumers' decisions for each draw of types and taste shocks. To solve this issue, we estimate the model using the importance-sampling method proposed in Ackerberg (2009). We rewrite the likelihood function by multiplying and dividing individual likelihoods by some density function $g\left(\theta_{i}\right)$, which we term proposal density:

$$
L(D \mid \Omega, p)=\prod_{i=1}^{N} \int\left(\prod_{t=1}^{T_{i}} L_{i t}\left(D_{i t} \mid \theta_{i}, p_{i t}\right) \cdot \frac{f\left(\theta_{i} \mid \Omega\right)}{g\left(\theta_{i}\right)}\right) g\left(\theta_{i}\right) d \theta_{i} .
$$

Importantly, we choose the function $g\left(\theta_{i}\right)$ such that it does not depend on hyperparameters $\Omega$. This observation suggests that instead of drawing consumers' types from the original density $f\left(\theta_{i} \mid \Omega\right)$, we can take $N_{M}$ draws of types from the proposal density $g\left(\theta_{i}\right)$ and approximate the likelihood function with a simulated counterpart:

$$
\tilde{L}(D \mid \Omega, p)=\prod_{i=1}^{N} \frac{1}{N_{M}} \sum_{m=1}^{N_{M}}\left(\prod_{t=1}^{T_{i}} \tilde{L}_{i t}\left(D_{i t} \mid \theta_{i}^{m}, p_{i t}\right) \cdot \frac{f\left(\theta_{i}^{m} \mid \Omega\right)}{g\left(\theta_{i}^{m}\right)}\right) .
$$

Here $\theta_{i}^{m}$ denotes the $m$-th draw of types for consumer $i$, and $\tilde{L}_{i t}\left(D_{i t} \mid \theta_{i}^{m}, p_{i t}\right)$ is the simulated consumer and session-specific likelihood contribution:

$$
\tilde{L}_{i t}\left(D_{i t} \mid \theta_{i}^{m}, p_{i t}\right)=\frac{1}{N_{S}} \sum_{s=1}^{N_{S}} 1\left\{W_{i t}\left(\theta_{i}^{m}, \mu_{i t}^{s}, \varepsilon_{i t}^{s}, p_{i t}\right) \geq 0\right\} .
$$

In this expression, $\mu_{i t}^{s}$ and $\varepsilon_{i t}^{s}$ are draws of taste shocks. We can then estimate parameters $\Omega$ by maximizing the simulated expression $\tilde{L}(D \mid \Omega, p)$ in (22). Appendix E describes the details of this estimation procedure.

The importance-sampling approach has several key advantages over a frequency estimator. First, this approach significantly reduces the computational burden of estimation. The only part of the likelihood in (22) that depends on parameters $\Omega$ is the weights $f\left(\theta_{i}^{m} \mid \Omega\right) / g\left(\theta_{i}^{m}\right)$, which implies that we do not need to recompute the likelihood contributions $\tilde{L}_{i t}\left(D_{i t} \mid \theta_{i}^{m}, p_{i t}\right)$ for each new guess of parameters $\Omega$. Instead, we can precompute these likelihood contributions for the generated draws of types $\theta_{i}^{m}$ and taste shocks $\mu_{i t}$ and $\epsilon_{i t}$ and use these values during maximization. Precomputing likelihood contributions significantly reduces the computational burden and allows us to use a relatively large number of draws per consumer. In our application, we set $N_{S}=1000$ and $N_{M}=$ 1000 , which results in at least 100,000 simulation draws per consumer.

The second advantage of the importance sampling method is that the resulting objective function is smooth in parameters because the weights $f\left(\theta_{i}^{m} \mid \Omega\right) / g\left(\theta_{i}^{m}\right)$ in (22) are continuous and dif- 
ferentiable in $\Omega$. This smoothness allows us to use gradient-based optimization methods instead of slower derivative-free alternatives. ${ }^{22}$

One practical consideration is the choice of the proposal density $g\left(\theta_{i}\right)$. Ideally, we would choose the proposal density that coincides with the true density of types $\theta_{i}, f\left(\theta_{i} \mid \Omega_{0}\right)$. In practice we choose $g\left(\theta_{i}\right)=f\left(\theta_{i} \mid \tilde{\Omega}_{0}\right)$, where $\tilde{\Omega}_{0}$ are the initial values of parameters used as a starting point for our estimation. To obtain reasonable initial values, we choose $\tilde{\Omega}_{0}$ that roughly matches four types of moments to their data counterparts: purchase probabilities, probabilities of first searches, persistence in purchases, and the expected number of searched products per session.

\section{Data and Results}

\subsection{Data and Descriptive Statistics}

We use data from a large international chain of cosmetic stores. The data contains search and purchase activity of consumers in the Chinese online store of this chain. The layout of the store's website is similar to that of other online retailers. Figure 3 shows the typical layout of an online store that looks similar to the one we analyze. Consumers can enter search terms or use category tags or filters to narrow down the set of relevant products. They are then presented with a product list displaying basic information such as product names, prices, photos, and short descriptions. Consumers can then click on any product to visit the product page which contains a more detailed product description as well as customer reviews.

Our dataset includes detailed information on browsing and purchase activity of all website users during the whole year of 2014. We observe the time stamp of each page visit, types of visited pages (e.g., main page, product page, shopping cart), and identities of products described on each visited page. We also observe purchases made through the website. In addition, the data includes unique consumer identifiers which we use to track consumers over time and match their browsing activity to related purchases. ${ }^{23}$

We focus on the category of face masks. The category is suitable for our analysis because of several reasons. Since consumers purchase face masks regularly, we can observe many of them making repeat purchases. Observing repeated purchases and corresponding search sessions is crucial for our empirical strategy, because the panel dimension of the data helps us identify preference heterogeneity (see our argument in Section 4.3). Another advantage of choosing face masks is that most of this category's search and purchase activity is concentrated around a small number of products. To simplify estimation, we focus on the 10 most popular moisturizers, which account for

\footnotetext{
${ }^{22}$ An alternative approach to smoothing the objective function is to use a kernel-smoothed frequency estimator as in Honka (2014) and Honka and Chintagunta (2017); however, in our application, we found such an approach to work significantly slower than importance sampling as it requires recomputing likelihoods for each new guess of parameters. In Appendix G we use a simulation study to compare computational time of a kernel-smoothed frequency estimator and the importance sampling approach.

${ }^{23}$ Our data provider uses cookies to track the browsing and purchase activity of users over time. Each time a user logs in, the website ties browsing history from a given cookie to the account of this user. In our data, we observe anonymized identifiers of consumers that correspond to unique accounts.
} 

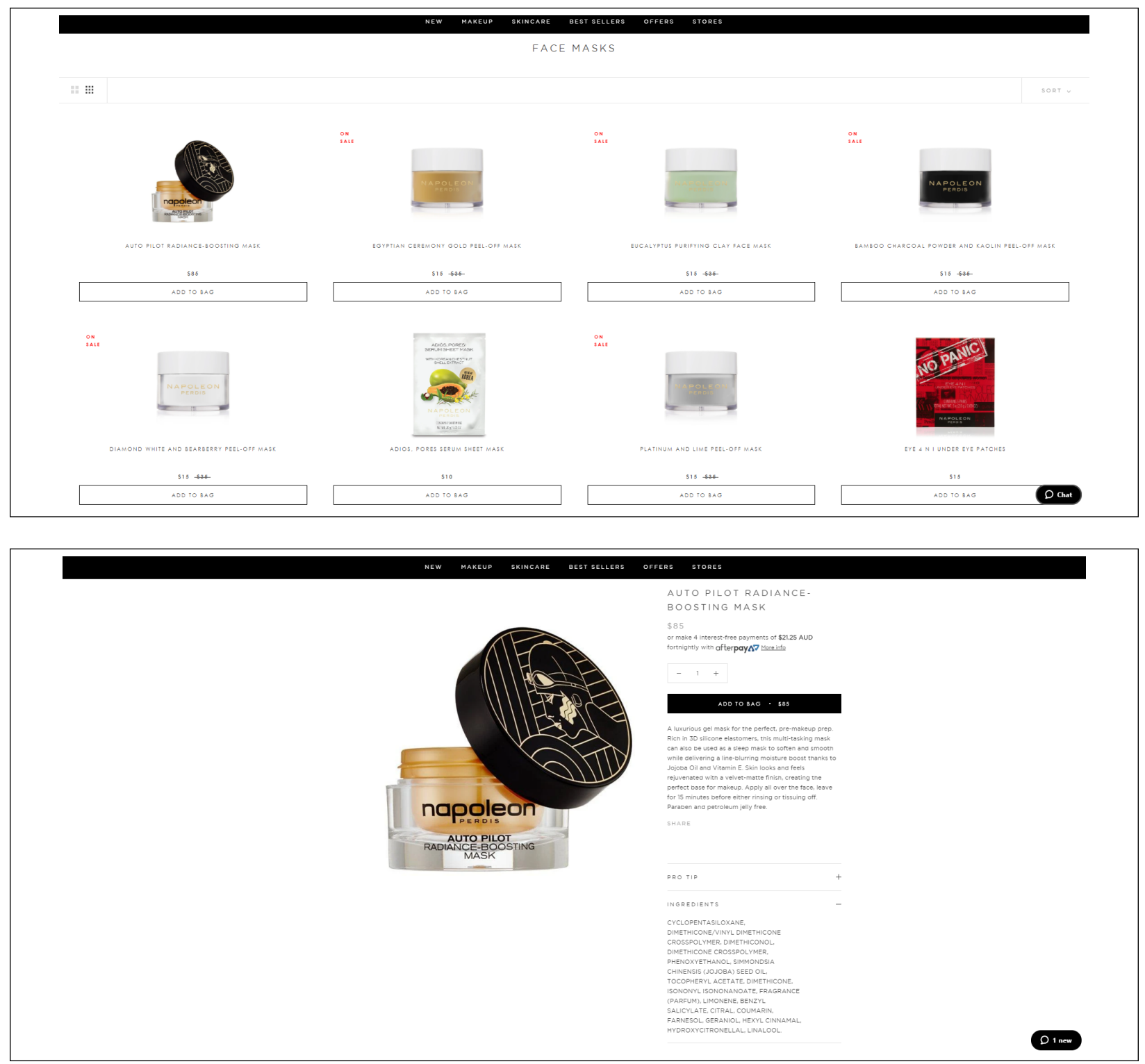

Figure 3: Examples of a category page and a product page. The two figures show examples of a category page (top figure) and a product page (bottom figure). To preserve the anonymity of our data provider, we show examples of webpages from another store that looks similar.

more than $50 \%$ of category sales.

We consider a consumer to be searching a given product if she visits the corresponding product page. ${ }^{24}$ We define each search session retrospectively from the purchase in which it ended.

\footnotetext{
${ }^{24}$ In principle, one could exclude very short searches because these might reflect navigational searches of consumers who already know which product they wish to purchase. We do not exclude any searches because short search durations are relatively rare in our data (only $15.5 \%$ of product page visits in our data are shorter than 15 seconds). Furthermore, the average duration of product page visits in single-product search sessions, which are more likely to be navigational, is similar to that in multi-product search sessions (the share of visits below 15 seconds is $18.5 \%$ in single-product sessions and $14.3 \%$ in multi-product sessions). We also note that the retailer's webpage allows consumers to purchase without visiting the product page, e.g., by clicking on the button "add to cart" that appears in the list of search results.
} 


\begin{tabular}{lcc} 
Panel A: Search Behavior & \\
\hline Average Number of Searches per Session & 2.42 \\
Number of Searches & 1 & $17.93 \%$ \\
Per Session & 2 & $41.18 \%$ \\
& 3 & $27.32 \%$ \\
& $\geq 4$ & $13.57 \%$
\end{tabular}

Panel B: Persistence

Purchase in $t=$ Purchase in $t-1 \quad 0.73$

First Search in $t=$ First Search in $\mathrm{t}-1 \quad 0.88$

$\frac{\text { Panel C: Product-Level }}{\text { Product } \quad \text { Brand }}$

$\begin{array}{cccc}\text { Avg. } & \text { Purch. } & \text { Search } & \text { Repeat } \\ \text { Price } & \text { Share } & \text { Share } & \text { Purch. } \\ \text { (Yuan) } & & & \text { Prob. }\end{array}$

\begin{tabular}{lccccc}
1 & Sephora & 63.0 & 0.297 & 0.237 & 0.762 \\
2 & Dr.Jart & 400.0 & 0.103 & 0.100 & 0.718 \\
3 & For Beloved One & 76.9 & 0.092 & 0.104 & 0.743 \\
4 & Benefit & 373.2 & 0.091 & 0.101 & 0.715 \\
5 & Caudalie & 420.7 & 0.040 & 0.037 & 0.696 \\
6 & Bliss & 488.1 & 0.101 & 0.110 & 0.724 \\
7 & Boscia & 60.2 & 0.085 & 0.104 & 0.721 \\
8 & Bliss & 158.0 & 0.048 & 0.058 & 0.712 \\
9 & Sephora & 230.0 & 0.022 & 0.024 & 0.702 \\
10 & Biotherm & 60.2 & 0.116 & 0.121 & 0.711 \\
\hline
\end{tabular}

Table 3: Descriptive Statistics for the Face Masks Category.

Specifically, the search session includes all searches that occurred within one week before a given purchase. ${ }^{25}$ Since it is difficult to define search sessions for consumers without purchases, we exclude these consumers from the data. For computational reasons, we only include consumers who made at least two purchases. The final estimation sample includes 11,540 search sessions conducted by 2,303 consumers. The average consumer in our data conducted five search sessions (i.e. made five purchases). Some consumers made as many as 35 purchases during the observation period.

Table 3 describes the main patterns of consumer behavior and presents some evidence of preference heterogeneity. Turning to search behavior (see Panel A of Table 3), we note that consumer search is limited. Consumers search on average only 2.42 products in a search session. Out of all search sessions, $17.9 \%$ finish after one search, $41.2 \%$ after two searches, and the remaining $40.9 \%$

\footnotetext{
${ }^{25}$ Over $80 \%$ of searches occur within one week before a purchase. When two purchases of the same consumer are less than seven days apart, we attribute only searches that occurred between two purchases to the later purchase.
} 
end after three or more searches.

Next, we investigate persistence in searches and purchases across different search sessions of the same consumer (Panel B of Table 3). Consumers tend to make persistent decisions: $73 \%$ of search sessions end in the same purchase as the previous search session. Moreover, search behavior exhibits a similar persistence, as $88 \%$ of the first searches are products that were also searched first during the previous session. The persistence in both purchases and searches suggests that consumers have substantially different preferences over the available products.

Finally, Panel $\mathrm{C}$ of Table 3 presents descriptive statistics at the product level. Prices vary substantially across products and range from 60.2 to 488.1 yuan (US\$8.6 and US\$69.8). Market shares are also significantly different, indicating some degree of vertical differentiation. We also report how frequently consumers make repeat purchases conditional on purchasing specific products in the past. Interestingly, even products that garner only a small market share tend to be purchased by consumers who have already purchased the same product in the past. For instance, the repeat purchase probabilities for the most popular product (30\% market share) and the least popular product $(2.2 \%$ market share) are remarkably similar: 0.76 versus 0.70 .

\subsection{Estimation Results}

Table 4 presents estimation results from our search model. The estimated parameters include means and standard deviations of product intercepts $\xi_{i j}$ for nine products (the intercept for the first product is normalized to zero) as well as parameters that determine the distribution of the price coefficients $\alpha_{i}$ and search costs $c_{i j}$. To ease interpretation, we report the implied means and standard deviations of price coefficients and search costs rather than the location and variance parameters of the corresponding log-normal distributions. Note that we cannot monetize estimated search costs, because they are identified only in relation to the normalized variance $\sigma_{\varepsilon}^{2}$ (see the discussion in section 5.1 and in Appendix C). ${ }^{26}$ We also present estimates from the perfect-information model in the last three columns of Table 4. This model assumes zero search costs for all consumers and products and is estimated using only purchase data, but it is otherwise identical to our search model.

Our analysis in section 3 suggests that ignoring search costs may lead to overestimation of preference heterogeneity. To establish whether this bias arises in our setting, we first need to make the estimated parameters from both models comparable. A natural way to compare estimates is to monetize them in both models. ${ }^{27}$ We report the monetized values of product intercepts and standard deviations in columns (3) and (6) of Table 4. According to the results, the perfectinformation model uniformly overestimates the variances of product intercepts. For the average product, the standard deviation is overestimated by 53\%. Furthermore, we find that estimated

\footnotetext{
${ }^{26}$ More specifically, all utility parameters are re-scaled by $1 / \sigma_{\mu}^{2}$, whereas search costs are re-scaled by $1 / \sigma_{\varepsilon}^{2}$. We can therefore not monetize the search cost estimate, but can monetize all other parameters that enter utility.

${ }^{27}$ Because the price coefficient is heterogeneous as well, we take draws from the distribution of estimated parameters and compute monetized preference parameters for each set of draws. We report the average value of monetized preference parameters across simulation draws.
} 


\begin{tabular}{|c|c|c|c|c|c|c|}
\hline & \multicolumn{3}{|c|}{ Search Model } & \multicolumn{3}{|c|}{ Perfect-Information Model } \\
\hline & Coefficients & $\begin{array}{l}\text { Standard } \\
\text { Errors }\end{array}$ & $\begin{array}{l}\text { Monetized } \\
\text { Parameters }\end{array}$ & Coefficients & $\begin{array}{c}\text { Standard } \\
\text { Errors }\end{array}$ & $\begin{array}{l}\text { Monetized } \\
\text { Parameters }\end{array}$ \\
\hline $\bar{\xi}_{2}$ & -4.38 & 1.52 & -154.0 & -4.76 & 0.17 & -199.6 \\
\hline $\bar{\xi}_{3}$ & -5.42 & 1.08 & -190.6 & -5.45 & 0.10 & -228.8 \\
\hline $\bar{\xi}_{4}$ & -9.54 & 1.89 & -335.6 & -6.47 & 0.15 & -271.4 \\
\hline $\bar{\xi}_{5}$ & -8.12 & 2.25 & -285.6 & -8.57 & 0.27 & -359.9 \\
\hline $\bar{\xi}_{6}$ & -7.01 & 0.75 & -246.7 & -5.87 & 0.17 & -246.8 \\
\hline $\bar{\xi}_{7}$ & -6.25 & 1.58 & -219.8 & -6.81 & 0.10 & -286.1 \\
\hline $\bar{\xi}_{8}$ & -7.84 & 0.95 & -275.8 & -8.65 & 0.21 & -363.2 \\
\hline $\bar{\xi}_{9}$ & -7.24 & 0.84 & -254.3 & -10.43 & 0.20 & -437.6 \\
\hline $\bar{\xi}_{10}$ & -4.74 & 0.67 & -166.9 & -4.58 & 0.07 & -192.6 \\
\hline$\sigma_{\xi_{2}}$ & 4.56 & 0.99 & 162.5 & 5.07 & 0.09 & 224.2 \\
\hline$\sigma_{\xi_{3}}$ & 4.68 & 1.44 & 166.8 & 5.07 & 0.11 & 215.2 \\
\hline$\sigma_{\xi_{4}}$ & 3.20 & 1.49 & 119.1 & 5.67 & 0.09 & 241.1 \\
\hline$\sigma_{\xi_{5}}$ & 4.08 & 1.04 & 147.5 & 5.39 & 0.10 & 230.2 \\
\hline$\sigma_{\xi_{6}}$ & 5.15 & 1.96 & 184.0 & 5.47 & 0.08 & 232.1 \\
\hline$\sigma_{\xi_{7}}$ & 4.34 & 0.88 & 155.6 & 5.55 & 0.07 & 235.8 \\
\hline$\sigma_{\xi_{8}}$ & 4.57 & 1.55 & 164.3 & 6.02 & 0.13 & 256.5 \\
\hline$\sigma_{\xi_{9}}$ & 4.72 & 1.31 & 169.4 & 6.87 & 0.10 & 293.4 \\
\hline$\sigma_{\xi_{10}}$ & 3.67 & 0.75 & 131.0 & 4.34 & 0.07 & 184.1 \\
\hline $\operatorname{Mean}(\alpha)$ & 0.0288 & 0.0087 & & 0.0240 & 0.0014 & \\
\hline $\operatorname{StdDev}(\alpha)$ & 0.0031 & 0.0015 & & 0.0024 & 0.0009 & \\
\hline $\operatorname{Mean}\left(c_{1}\right)$ & 0.0064 & 0.0044 & & & & \\
\hline $\operatorname{Mean}\left(c_{2}\right)$ & 0.0021 & 0.0015 & & & & \\
\hline $\operatorname{Mean}\left(c_{3}\right)$ & 0.0010 & 0.0008 & & & & \\
\hline $\operatorname{Mean}\left(c_{4}\right)$ & 0.0005 & 0.0002 & & & & \\
\hline $\operatorname{Mean}\left(c_{5}\right)$ & 0.0066 & 0.0021 & & & & \\
\hline $\operatorname{Mean}\left(c_{6}\right)$ & 0.0012 & 0.0017 & & & & \\
\hline $\operatorname{Mean}\left(c_{7}\right)$ & 0.0015 & 0.0004 & & & & \\
\hline $\operatorname{Mean}\left(c_{8}\right)$ & 0.0010 & 0.0031 & & & & \\
\hline $\operatorname{Mean}\left(c_{9}\right)$ & 0.0093 & 0.0065 & & & & \\
\hline $\operatorname{Mean}\left(c_{10}\right)$ & 0.0019 & 0.0001 & & & & \\
\hline $\operatorname{StdDev}(c)$ & 0.0003 & 0.0002 & & & & \\
\hline LogLikelihood & -37623.5 & & & -22237.1 & & \\
\hline AIC & 75739.0 & & & 44514.7 & & \\
\hline $\mathrm{BIC}$ & 75865.3 & & & 44629.1 & & \\
\hline
\end{tabular}

Table 4: Estimation Results from the Search Model and the Perfect Information Model. The unit of observation is a search session of a specific consumer. The sample contains 2,303 consumers and 11,540 search sessions ( 5 search sessions per consumer on average). Monetized intercepts are reported in yuans net of price effects. The intercepts for product 1 are normalized for all consumers. Fit statistics are not directly comparable for the two models because their estimation is based on different datasets: the model in columns 1-3 uses search data which is not used in the perfect-information model in columns 4-6. 


\begin{tabular}{cccccc}
\hline \multicolumn{2}{c}{ I. Purchase Probabilities } & \multicolumn{3}{c}{$\begin{array}{c}\text { II. Search Probabilities } \\
\text { (Based on First Searches) }\end{array}$} \\
\hline Product & Data & Predicted & Product & Data \\
\hline 1 & 0.295 & 0.301 & 1 & 0.303 & 0.232 \\
2 & 0.099 & 0.107 & 2 & 0.115 & 0.104 \\
3 & 0.081 & 0.104 & 3 & 0.095 & 0.111 \\
4 & 0.086 & 0.023 & 4 & 0.088 & 0.031 \\
5 & 0.057 & 0.040 & 5 & 0.063 & 0.033 \\
6 & 0.098 & 0.083 & 6 & 0.099 & 0.103 \\
7 & 0.093 & 0.105 & 7 & 0.095 & 0.119 \\
8 & 0.051 & 0.077 & 8 & 0.060 & 0.093 \\
9 & 0.026 & 0.047 & 9 & 0.033 & 0.048 \\
10 & 0.114 & 0.114 & 10 & 0.049 & 0.126 \\
\hline \multicolumn{7}{c}{} & III. Persistence & & IV. Number of Searches \\
\hline Purchases & Data & Predicted & & Data & Predicted \\
\hline Sirst Searches & 0.734 & 0.666 & & 2.47 & 2.16 \\
\hline
\end{tabular}

Table 5: Out-of-Sample Fit of the Search Model. For the out-of-sample validation exercise, we split our sample of 2,303 consumers into two parts, allocating $80 \%$ of them to the training sample (1,842 consumers) and $20 \%$ to the holdout sample (461 consumers). We then estimate the model on the training sample and use the estimated model to predict several key moments. This table compares these predicted moments to their counterparts observed in the holdout sample.

mean intercepts are almost uniformly biased away from zero.

We regards to search cost estimates, we find that estimated search costs differ somewhat across products. As discussed in Section 3.2, strong asymmetries in search costs across products could potentially lead to an underestimation of preference heterogeneity, whereas relatively similar search costs typically imply that heterogeneity is overestimated when we ignore search. Our empirical application falls into the latter category: we find that omitting costly search from the model leads to an overestimation of preference heterogeneity for all products. To further explore the role of asymmetric search costs, we also estimate a restricted model with common search costs across products. We report the results from this specification in Table 6 next to the results from our baseline model with product-specific search costs. We find that forcing search costs to be identical across products still leads to an overestimation of preference heterogeneity relative to the perfect information model.

\subsection{Out-of-sample Fit}

We examine model fit by comparing several key predictions to their empirical counterparts. To this end, we split the sample of 2,303 consumers into a $80 \%$ training sample of 1842 consumers and a $20 \%$ holdout sample of 461 consumers. Table 5 reports the simulated predictions and their empirical counterparts for the holdout sample. We first evaluate fit in terms of purchase and first search probabilities in panels I and II and find that the model predicts the held-out data reasonably 
well. Similarly, it predicts the average number of searches reasonably well. The model does slightly worse in terms of fitting persistence in searches and purchases: both measures of persistence are underestimated. We note the model contains no parameters that specifically cater to matching persistence, such as, for example, switching costs. Hence, the predicted degree of persistence is entirely driven by preference heterogeneity and search costs.

We also report measures of in-sample statistical fit at the bottom of Table 4. However, these measures are not directly comparable between the models with and without search. This is because we are not merely fitting different models to the same data, but we are also using more data and a different likelihood function in the model with costly search. While in the search model we are maximizing the joint-likelihood of search and purchase decisions, in the model without search we are maximizing the likelihood of purchase decisions.

\subsection{Extension: Persistent Information Across Search Sessions}

In our baseline model, we assume that taste shocks $\varepsilon_{i j t}$ are independent across search sessions. Therefore, any information a consumer obtains about a product in a given search session does not predict utility from the same product in future sessions. However, it is possible that consumers retain some information from one session to another. To allow for this possibility, we now consider an extension where we allow for partial correlation in information, which nests our baseline model as a special case when the correlation is set to zero. There are two main reasons why information might be correlated over time, but only partially so. First, new information might become available by the time a consumer returns for another search session. This new information might take the form of updated product page content which features new customer ratings and reviews, additional photos, or changes in the product description. Second, partial correlation may arise if consumers remember some product features they have learned in previous sessions but forget others, thus partially retaining information acquired during prior search.

To model partial correlation of taste shocks across search sessions, we assume that shocks $\varepsilon_{i j t}$ follow an MA(1) process so that

$$
\varepsilon_{i j t}=\omega_{i j t}+\rho \omega_{i j t-1}
$$

where $\omega_{i j t}$ follows a standard normal distribution and is iid across consumers, products, and search sessions. Upon searching product $j$ in period $t$, the consumer $i$ observes both the taste shock $\varepsilon_{i j t}$ and the transitory shock $\omega_{i j t}$. As before, we assume that consumers have rational expectations consistent with the true distribution of taste shocks. These assumptions imply that consumer's beliefs at the beginning of search session $t$ can be summarized as follows: ${ }^{28}$

\footnotetext{
${ }^{28}$ If the consumer did not search a given product in the previous time period, she does not know the realization of $\omega_{i j t-1}$; therefore, from her perspective, $E\left(\varepsilon_{i j t}\right)=0$ and $\operatorname{Var}\left(\varepsilon_{i j t}\right)=\operatorname{Var}\left(\omega_{i j t}\right)+\rho^{2} \operatorname{Var}\left(\omega_{i j t-1}\right)=\sigma_{\omega}^{2} \cdot\left(1+\rho^{2}\right)$. On the other hand, if the consumer does know the realization of $\omega_{i j t-1}$, it follows that $E\left(\varepsilon_{i j t} \mid \omega_{i j t-1}\right)=\rho \omega_{i j t-1}$ and $\operatorname{Var}\left(\varepsilon_{i j t} \mid \omega_{i j t-1}\right)=\operatorname{Var}\left(\omega_{i j t}\right)+\rho^{2} \cdot 0=\sigma_{\omega}^{2}$.
} 


\begin{tabular}{|c|c|c|c|c|c|c|c|c|c|}
\hline & \multicolumn{3}{|c|}{ Main Specification } & \multicolumn{3}{|c|}{ Common Search Costs } & \multicolumn{3}{|c|}{$\frac{\text { Persistence in }}{\text { Information }}$} \\
\hline & Coef. & $\begin{array}{l}\text { Stand. } \\
\text { Errors }\end{array}$ & $\begin{array}{l}\text { Monet. } \\
\text { Param. }\end{array}$ & Coef. & $\begin{array}{l}\text { Stand. } \\
\text { Errors }\end{array}$ & $\begin{array}{l}\text { Monet. } \\
\text { Param. }\end{array}$ & Coef. & $\begin{array}{l}\text { Stand. } \\
\text { Errors }\end{array}$ & $\begin{array}{l}\text { Monet. } \\
\text { Param. }\end{array}$ \\
\hline $\bar{\xi}_{2}$ & -4.38 & 1.52 & -154.0 & -4.48 & 1.56 & -128.3 & -3.14 & 1.33 & -117.0 \\
\hline $\bar{\xi}_{3}$ & -5.42 & 1.08 & -190.6 & -4.68 & 0.74 & -134.3 & -5.69 & 0.94 & -211.5 \\
\hline $\bar{\xi}_{4}$ & -9.54 & 1.89 & -335.6 & -9.68 & 1.95 & -277.4 & -9.47 & 4.13 & -351.7 \\
\hline $\bar{\xi}_{5}$ & -8.12 & 2.25 & -285.6 & -7.95 & 1.88 & -227.9 & -7.83 & 3.12 & -291.0 \\
\hline $\bar{\xi}_{6}$ & -7.01 & 0.75 & -246.7 & -6.76 & 0.69 & -193.9 & -6.35 & 0.06 & -236.0 \\
\hline $\bar{\xi}_{7}$ & -6.25 & 1.58 & -219.8 & -6.63 & 2.22 & -190.2 & -6.11 & 2.57 & -227.3 \\
\hline $\bar{\xi}_{8}$ & -7.84 & 0.95 & -275.8 & -7.47 & 1.53 & -214.2 & -6.97 & 1.47 & -259.1 \\
\hline $\bar{\xi}_{9}$ & -7.24 & 0.84 & -254.3 & -8.52 & 0.76 & -244.1 & -8.90 & 0.46 & -330.8 \\
\hline $\bar{\xi}_{10}$ & -4.74 & 0.67 & -166.9 & -4.28 & 0.91 & -122.8 & -4.58 & 0.08 & -170.4 \\
\hline$\sigma_{\xi_{2}}$ & 4.56 & 0.99 & 162.5 & 4.97 & 0.90 & 144.0 & 3.73 & 0.79 & 138.7 \\
\hline$\sigma_{\xi_{3}}$ & 4.68 & 1.44 & 166.8 & 3.91 & 1.26 & 113.6 & 3.79 & 1.49 & 140.9 \\
\hline$\sigma_{\xi_{4}}$ & 3.20 & 1.49 & 119.1 & 3.17 & 1.57 & 95.1 & 3.10 & 1.06 & 115.2 \\
\hline$\sigma_{\xi_{5}}$ & 4.08 & 1.04 & 147.5 & 4.08 & 1.34 & 119.5 & 3.67 & 1.28 & 136.6 \\
\hline$\sigma_{\xi_{6}}$ & 5.15 & 1.96 & 184.0 & 5.32 & 2.53 & 154.4 & 4.65 & 3.51 & 173.2 \\
\hline$\sigma_{\xi_{7}}$ & 4.34 & 0.88 & 155.6 & 4.71 & 0.79 & 136.8 & 4.49 & 1.25 & 166.8 \\
\hline$\sigma_{\xi_{8}}$ & 4.57 & 1.55 & 164.3 & 4.45 & 1.57 & 129.9 & 4.89 & 1.47 & 181.7 \\
\hline$\sigma_{\xi_{9}}$ & 4.72 & 1.31 & 169.4 & 5.03 & 1.39 & 146.9 & 4.81 & 1.65 & 178.8 \\
\hline$\sigma_{\xi_{10}}$ & 3.67 & 0.75 & 131.0 & 3.43 & 0.69 & 99.4 & 3.70 & 0.86 & 137.3 \\
\hline $\operatorname{Mean}(\alpha)$ & 0.0288 & 0.0087 & & 0.0352 & 0.0065 & & 0.0269 & 0.0109 & \\
\hline $\operatorname{StdDev}(\alpha)$ & 0.0031 & 0.0015 & & 0.0034 & 0.0009 & & 0.0020 & 0.0062 & \\
\hline $\operatorname{Mean}\left(c_{1}\right)$ & 0.0064 & 0.0044 & & & & & & & \\
\hline $\operatorname{Mean}\left(c_{2}\right)$ & 0.0021 & 0.0015 & & & & & & & \\
\hline $\operatorname{Mean}\left(c_{3}\right)$ & 0.0010 & 0.0008 & & & & & & & \\
\hline Mean $\left(c_{4}\right)$ & 0.0005 & 0.0002 & & & & & & & \\
\hline Mean $\left(c_{5}\right)$ & 0.0066 & 0.0021 & & & & & & & \\
\hline $\operatorname{Mean}\left(c_{6}\right)$ & 0.0012 & 0.0017 & & & & & & & \\
\hline $\operatorname{Mean}\left(c_{7}\right)$ & 0.0015 & 0.0004 & & & & & & & \\
\hline Mean $\left(c_{8}\right)$ & 0.0010 & 0.0031 & & & & & & & \\
\hline Mean $\left(c_{9}\right)$ & 0.0093 & 0.0065 & & & & & & & \\
\hline Mean $\left(c_{10}\right)$ & 0.0019 & 0.0001 & & & & & & & \\
\hline $\operatorname{Mean}(c)$ & & & & 0.0032 & 0.0020 & & 0.0028 & 0.0060 & \\
\hline $\operatorname{StdDev}(c)$ & 0.0003 & 0.0002 & & 0.0003 & 0.0001 & & 0.0003 & 0.0001 & \\
\hline$\rho$ & & & & & & & 0.3 & & \\
\hline LogLikelihood & & -37623.5 & & & -37950.9 & & & -37750.2 & \\
\hline AIC & & 75739.0 & & & 76281.7 & & & 76023.3 & \\
\hline $\mathrm{BIC}$ & & 75865.3 & & & 76291.4 & & & 76112.0 & \\
\hline
\end{tabular}

Table 6: Alternative Specifications: Common Search Costs Across Products and Persistence in Information. The unit of observation is a search session of a specific consumer. The sample contains 2,303 consumers and 11,540 search sessions. 


$$
\begin{aligned}
& \varepsilon_{i j t} \sim N\left(0, \sigma_{\omega}^{2} \cdot\left(1+\rho^{2}\right)\right) \text { if product } j \text { was NOT searched in } t-1 \\
& N\left(\rho \omega_{i j t-1}, \sigma_{\omega}^{2}\right) \quad \text { if product } j \text { was searched in } t-1 \text {, }
\end{aligned}
$$

Hence, the information obtained when searching a product in a given search session partly carries over to the next search session. ${ }^{29}$ The extended model leads to different post-search taste shock variances across products. However, within a search session, taste shocks are still independently distributed across products; therefore, the optimal search rule can be characterized using Weitzman's threshold rule described earlier. In Appendix F, we re-write the likelihood function to accommodate correlated taste shocks and discuss estimation details for this extended model.

The new parameter $\rho$ introduces correlation in taste shocks across sessions, thus potentially increasing persistence in what consumers search and purchase. Since this effect is similar to that of increasing preference heterogeneity, one may ask whether it is possible to identify the correlation parameter $\rho$ separately from the preference heterogeneity parameters $\sigma_{\xi_{j}}$. To see why these parameters can be separately identified, note that the correlation $\rho$ introduces "structural" state dependence, i.e, persistence in choices that is not driven by time-invariant differences in preferences and instead driven by a causal impact of past searches on current decisions. Therefore, if a temporary price discount makes a consumer more likely to search and purchase a given product, this induced choice will generate a causal effect on decisions made in the next session. This is in contrast to a model without correlation $(\rho=0)$ in which decisions in the previous search session have no direct impact on future decisions.

This identification argument is similar in spirit to the arguments developed in the literature on switching costs (Dubé, Hitsch, and Rossi (2010)). The main difference from their argument, however, is that in our model, the structural dependence in choices manifests itself in both search and purchase decisions. If a consumer searched and purchased a product in the past, this suggests she received a favorable draw of shock $\varepsilon_{i j t}$ for the product and should be more likely to search and purchase this product in the current session. Instead, if a consumer searched but did not purchase a product, she likely received an unfavorable draw $\varepsilon_{i j t}$ and should therefore be less likely to search and purchase this product in the following session. Hence, we can identify the correlation $\rho$ from the degree to which consumers' search and purchase decisions are affected by which products they searched and purchased in the previous search session. The results from this extension are reported in the rightmost columns of Table 6 . We find estimated correlation to be relatively low with $\hat{\rho}=0.3 .^{30}$ Most importantly, the estimates of preference heterogeneity do not significantly

\footnotetext{
${ }^{29}$ We assume that consumers do not anticipate how their current search decisions affect their information set in future search sessions. Given that search sessions in our data tend to be relatively spread out in time, we consider this to be a reasonable assumption.

${ }^{30}$ We obtain the estimate of $\hat{\rho}=0.3$ using grid search (see Appendix F for details) but do not compute a standard error for this estimate. We proceed in this way because the model with correlated information is computational burdensome to estimate, which prevents us from using a bootstrap procedure to calculate standard errors. For computational reasons we also constrain search costs not to vary across products when estimating this model extension.
} 


\begin{tabular}{|c|c|c|}
\hline & \multicolumn{2}{|c|}{ Own-price elasticities } \\
\hline & & Perfect \\
\hline & Search & Information \\
\hline Product & Model & Model \\
\hline 1 & -0.511 & -0.492 \\
\hline 2 & -3.156 & -2.536 \\
\hline 3 & -0.737 & -0.581 \\
\hline 4 & -5.862 & -2.383 \\
\hline 5 & -4.816 & -3.175 \\
\hline 6 & -3.996 & -3.018 \\
\hline 7 & -0.673 & -0.444 \\
\hline 8 & -1.825 & -1.109 \\
\hline 9 & -2.359 & -1.519 \\
\hline 10 & -0.724 & -0.502 \\
\hline Average & -2.506 & -1.576 \\
\hline
\end{tabular}

Table 7: Estimated Own-price Elasticities. The table compares own-price elasticities predicted by two models: the search model and the perfect information model. All elasticities are computed at the current prices observed in the data.

change when we allow for correlation in taste shocks. Therefore, while this extension provides a more flexible modeling framework, allowing for correlated information does not change the main conclusions regarding the estimates of preferences heterogeneity.

\section{Personalized Pricing}

\subsection{Price Elasticities and Markups}

The bias in heterogeneity estimates may affect our inference about price elasticities and the market power of firms. Because we overestimate the degree to which preferences are different across consumers, we mistakenly conclude that most consumers have strong preferences for certain products and therefore respond little to price changes. ${ }^{31}$ As a result, we may underestimate demand elasticities and incorrectly conclude that competition among firms is mild.

We illustrate this point in Table 7 where we report price elasticities from the search and perfectinformation models. Consistent with the logic above, the perfect-information model underestimates the absolute values of own-price elasticities for all ten products. This bias in elasticities, in turn, affects our inference about markups of firms. Assuming Bertrand-Nash pricing, we obtain that the perfect-information model implies an average markup of 245.0 yuan, whereas the search model predicts an average markup of only 205.8 yuan. Therefore, by ignoring search, we overestimate markups of firms by $19 \%$. Overall, our results suggest that, to correctly understand the competitive environment, one needs to account for search frictions and the way they affect firms' pricing

\footnotetext{
${ }^{31}$ Several authors have emphasized that higher dispersion of consumer preferences often leads to less elastic demand (Anderson and Renault, 1999; Zhou, 2017).
} 
strategies. If a researcher incorrectly infers from high choice persistence that products are highly differentiated, she will tend to underestimate how strongly firms compete with each other.

\subsection{Personalized Prices}

We now consider the implications of our results for personalized marketing. In general, how much a firm can benefit from personalizing its marketing activities critically depends on its ability to assess the heterogeneity of consumer preferences. Therefore, if preference heterogeneity is overestimated, a firm may overestimate the scope for personalization and may engage in suboptimal targeting. To illustrate this point, we consider the example of personalized pricing in which for a given product, a firm sets different prices based on consumers' search and purchase histories.

We first develop a general framework to analyze personalized pricing under different models of consumer behavior and for different consumer histories. To this end, we start by deriving the profit function of the firm for a given consumer $i$ (the expressions below omit index $i$ to avoid clutter). Suppose the firm observes consumer $i$ 's history of searches and purchases. The history consists of all products searched in previous sessions, the order in which these products were searched, and purchase decisions. Let $H$ denote this history and let $p^{H}=\left\{p_{1 t}, \ldots, p_{J t}\right\}_{t=1}^{T}$ denote prices the consumer faced in these previous search sessions, where $T$ is the number of search sessions the consumer conducted in the past. The firm infers consumer type $\theta$ from history $H$ and $p^{H}$. Assuming the prior distribution of the firm coincides with the true distribution of types $f\left(\theta \mid \Omega_{0}\right)$, the posterior distribution of types $\theta$ given history $H$ follows Bayes' rule: ${ }^{32}$

$$
f\left(\theta \mid H, p^{H}, \Omega_{0}\right)=\frac{L\left(H \mid p^{H}, \theta\right) f\left(\theta \mid \Omega_{0}\right)}{\int L\left(H \mid p^{H}, \theta\right) d F\left(\theta \mid \Omega_{0}\right)},
$$

where $L\left(H \mid p^{H}, \theta\right)$ is the likelihood of observing history $H$ given historical prices $p^{H}$ and type $\theta$, and $\Omega_{0}$ is a vector of the true parameter values. Given this posterior distribution of types, $f\left(\theta \mid H, p^{H}, \Omega_{0}\right)$, the firm chooses the price $p_{j}$ for product $j$ to maximize its expected total profit

$$
\Pi\left(p_{j}, H\right)=\sum_{k \in \mathcal{F}} \int\left(p_{k}-m c_{k}\right) \operatorname{Pr}(y=k \mid \theta) f\left(\theta \mid H, p^{H}, \Omega\right) g(\Omega) d \theta d \Omega .
$$

where $p_{k}$ is the price of product $k, m c_{k}$ denotes marginal costs, $\mathcal{F}$ describes the set of all products sold by the firm, and $\operatorname{Pr}(y=k \mid \theta)$ is the purchase probability conditional on the consumer's type.

The firm faces two sources of uncertainty: about the type $\theta$, which is captured by the posterior density $f\left(\theta \mid H, p^{H}, \Omega\right)$, and about hyperparameters $\Omega$, which is reflected in the density $g(\Omega)$. We approximate posterior beliefs about type $\theta$ by taking draws from the posterior distribution $f\left(\theta \mid H, p^{H}, \Omega\right)$. Similarly, we approximate the distribution $g(\Omega)$ using the asymptotic distribution of the maximum likelihood estimates $\hat{\Omega}$ (see Appendix $\mathrm{H}$ for details). In addition, we infer

\footnotetext{
${ }^{32}$ Our estimation approach recovers the parameters that govern the distribution of consumer types but does not explicitly recover the preferences and search costs of individual consumers. Therefore, we require this additional step to infer individual-level parameters based consumers' search and purchase histories.
} 


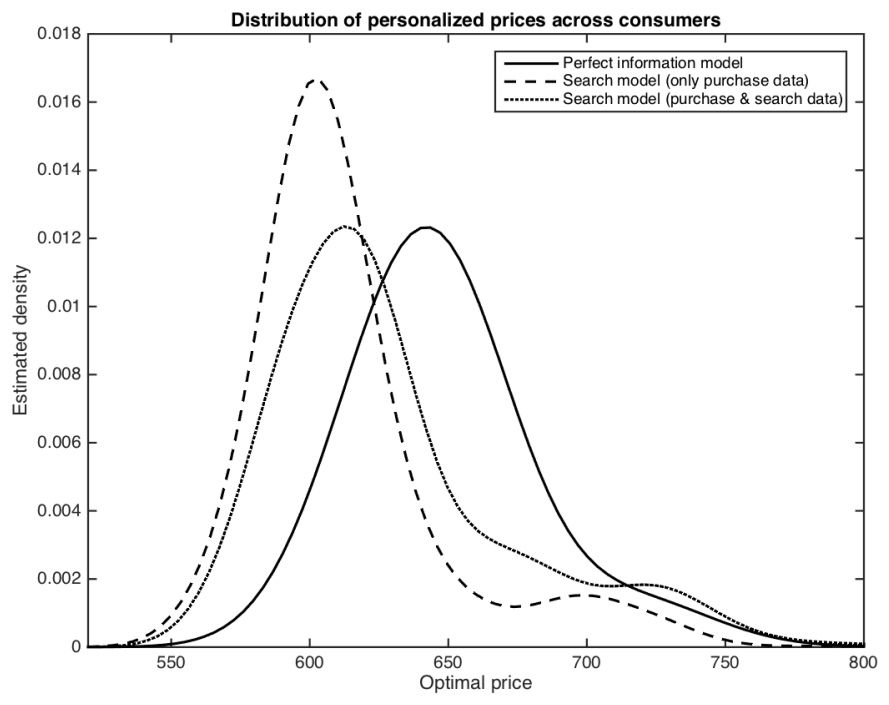

Figure 4: Distribution of Personalized Prices. The graph plots the kernel densities of personalized prices under three scenarios: (a) perfect-information model (solid line), (b) search model in which types are inferred based only on purchase data (dashed line), and (c) search model in which types are inferred from both search and purchase data (dotted line).

marginal costs $m c_{j}$ by assuming that the current prices arise from a Nash Equilibrium. When inferring marginal costs, we assume firms set their prices based on the perfect-information demand model.

To evaluate profits from different pricing regimes, we use the expected profit function from the estimated search model. By construction, this profit function is maximized when the seller sets personalized prices based on the search model and infers individual preferences from complete histories of past searches and purchases. Below, we derive optimal personalized prices separately for each product, assuming the firm sets prices for product $j$ while holding fixed the (uniform) prices of other products. This simplifying assumption allows us to abstract away from potential equilibrium effects and helps us focus on assessing the scope for targeting.

We first compute personalized prices based on the perfect-information model. Specifically, we use estimates from the perfect information model and infer types $\theta$ only from purchase histories $H=\left\{y_{t}\right\}_{t=1}^{T}$. Figure 4 shows the resulting distribution of personalized prices for one product in our sample. ${ }^{33}$ To quantify losses from setting prices based on the model that ignores search, we insert obtained personalized prices into the profit function from the search model. We report the resulting change in profits in Table 8 . The results show that targeting based on the model that ignores search reduces expected profits on average by $0.6 \%$ relative to uniform pricing.

Next, we analyze personalized prices derived from the search model. To isolate the effect of switching to a different model of consumer behavior, we only allow the firm to set prices based

\footnotetext{
${ }^{33}$ The graph refers to product 5 , which has an average price of $\bar{p}_{5}=649.9$ during our sample period, and for which the inferred marginal cost equals $\hat{m} c_{5}=540.2$.
} 


\begin{tabular}{cccc}
\hline \multicolumn{3}{c}{ Profit increase from personalization (percent) } \\
\hline Product & $\begin{array}{c}\text { Perfect } \\
\text { Information } \\
\text { Model }\end{array}$ & $\begin{array}{c}\text { Search Model } \\
\text { (Purchase Data } \\
\text { Only) }\end{array}$ & $\begin{array}{c}\text { Search Model } \\
\text { (Purchase and } \\
\text { Search Data) }\end{array}$ \\
\hline 1 & -3.66 & 3.38 & 7.24 \\
2 & 1.31 & 9.88 & 15.30 \\
3 & 0.54 & 2.23 & 6.28 \\
4 & -4.28 & 0.25 & 11.45 \\
5 & -1.52 & 7.40 & 10.90 \\
6 & 1.43 & 2.30 & 9.10 \\
7 & -0.42 & 0.65 & 5.90 \\
8 & -0.57 & 5.74 & 11.91 \\
9 & 1.06 & 4.20 & 9.80 \\
10 & 0.29 & 0.64 & 2.77 \\
\hline Average & -0.58 & 3.67 & 9.07 \\
\hline
\end{tabular}

Table 8: Expected Gains from Personalized Pricing under Different Models and Consumer Histories. The gains from charging personalized prices are computed relative to the expected profits from optimal uniform prices. The table reports gains for three scenarios: (a) pricing based on the perfect-information model, (b) pricing based on the search model with type inference based only on purchase data, and (c) pricing based on the search model with type inference based on both search and purchase data.

on purchase histories as in the previous exercise (i.e, the firm is not allowed to price discriminate based on search histories). The results in Figure 4 suggest the distribution of personalized prices has a similar shape as the one generated by the perfect information model but is shifted to the left. Consistent with the discussion in the previous section, this shift occurs because the search model predicts higher absolute values of own-price elasticities, thus suggesting to set lower optimal prices than those obtained from the perfect information model. Furthermore, these prices have a lower variance than those generated by the perfect information model. This difference arises because the perfect information model overestimates preference heterogeneity, thus overestimating the scope for targeting. Overall, the results in the second column of Table 8 suggest that setting personalized prices based on the search model and only purchase histories increases profits for the average product by $3.7 \%$ relative to uniform pricing.

Finally, we derive optimal personalized prices based on the search model but now allow the firm to set prices based on both purchase and search histories. Adding search data to consumers' histories leads to more precise inference about types $\theta$, thus generating higher profits from personalized pricing. Specifically, our results suggest that, compared to uniform pricing, the expected profits for the average product increase by $9.1 \%$.

In summary, firms gain little by charging personalized prices based on the perfect information model. In fact, setting prices based on this model reduces profits from the average product by $0.6 \%$. Some products loose even more: for example, profits from product 4 decrease by $4.3 \%$ from personalization based on the perfect information model. By contrast, firms' profits significantly 
increase when they explicitly account for costly search and use search data to infer consumers' types. Compared to uniform pricing, profits for the average product increase by $3.7 \%$ when firms set prices based on the search model but only use purchase histories for targeting. Profits increase by $9.1 \%$ when firms rely on the search model and personalize based on both search and purchase histories.

\section{Conclusion}

This paper studies the estimation of preference heterogeneity in markets where consumers are imperfectly informed and engage in costly search. We show that in the typical search model, ignoring search costs leads to an overestimation of preference heterogeneity. In our empirical exercise, we find that by ignoring search costs, we overestimate standard deviations of product intercepts by $53 \%$. This bias has important consequences for optimal price setting. When ignoring search frictions, we underestimate own-price elasticities and overestimate firms' markups. Hence, when search is omitted from the analysis, we underestimate the intensity of competition between firms. In addition, personalized prices generated based on a perfect-information model are more dispersed than prices computed based on the estimated search model. As a result, personalized

prices computed under the incorrect assumption of perfect information reduce expected profits by $0.6 \%$. Personalizing prices based on the search model, by contrast, increases expected profits on average by $9.1 \%$. Thus, ignoring costly search costs leads to suboptimal pricing strategies. 


\section{References}

AckerberG, D. A. (2009): "A new use of importance sampling to reduce computational burden in simulation estimation," QME, 7(4), 343-376.

Allenby, G. M., And P. E. Rossi (1999): "Marketing Models of Consumer Heterogeneity," Journal of Econometrics, 89(1), 57-78.

Anderson, S. P., And R. Renault (1999): "Pricing, product diversity, and search costs: A Bertrand-Chamberlin-Diamond model," The RAND Journal of Economics, pp. 719-735.

Chade, H., and L. Smith (2006): "Simultaneous search," Econometrica, 74(5), 1293-1307.

Chen, Y., And S. Yao (2017): "Sequential Search with Refinement: Model and Application with Click-stream Data," Management Science, 635(12), 4345-4365.

Chintagunta, P. K., D. C. Jain, and N. J. Vilcassim (1991): "Investigating Heterogeneity in Brand Preferences in Logit Models for Panel Data," Journal of Marketing Research, 28(4), $417-428$.

De Los Santos, B. I., A. Hortacsu, and M. Wildenbeest (2012): "Testing Models of Consumer Search using Data on Web Browsing and Purchasing Behavior," American Economic Review, 102(6), 2955-2980.

Dubé, J.-P., G. J. Hitsch, And P. E. Rossi (2009): "Do Switching Costs Make Markets Less Competitive?," Journal of Marketing Research, 46(4), 435-445.

- (2010): "State Dependence and Alternative Explanations for Consumer Inertia," The RAND Journal of Economics, 41(3), 417-445.

Dubé, J.-P., G. J. Hitsch, P. E. Rossi, And M. A. Vitorino (2008): "Category Pricing with State-Dependent Utility," Marketing Science, 27(3), 417-429.

Elberg, A., P. M. Gardete, R. Macera, and C. Noton (2017): "Dynamic Effects of Price Promotions: Field Evidence, Consumer Search, and Supply-Side Implications," .

Giulietti, M., M. Waterson, and M. Wildenbeest (2014): "Estimation of search frictions in the British electricity market," The Journal of Industrial Economics, 62(4), 555-590.

HonkA, E. (2014): "Quantifying search and switching costs in the US auto insurance industry," The RAND Journal of Economics, 45(4), 847-884.

Honka, E., And P. K. Chintagunta (2017): "Simultaneous or sequential? search strategies in the us auto insurance industry," Marketing Science, 36(1), 21-42.

Kim, J. B., P. Albuquerque, and B. J. Bronnenberg (2010): "Online Demand under Limited Consumer Search," Marketing Science, 29(6), 1001-1023.

Koulayev, S. (2014): "Search for Differentiated Products: Identification and Estimation," The RAND Journal of Economics, 45(3), 553-575.

Rossi, P. E., And G. M. Allenby (1993): "A bayesian approach to estimating household parameters," Journal of Marketing Research, 30(2), 171-182. 
Rossi, P. E., R. E. McCulloch, and G. M. Allenby (1996): "The Value of Purchase History Data in Target Marketing," Marketing Science, 15(4), 321-340.

SeILER, S. (2013): "The Impact of Search Costs on Consumer Behavior: A Dynamic Approach," Quantitative Marketing and Economics, 11(2), 155-203.

Ursu, R. M. (2018): "The Power of Rankings: Quantifying the Effect of Rankings on Online Consumer Search and Purchase Decisions," Marketing Science, 37(4), 530-552.

Weitzman, M. L. (1979): "Optimal Search for the Best Alternative," Econometrica, 47(3), 641654.

Yavorsky, D., E. Honka, And K. Chen (2020): "Consumer Search in the U.S. Auto Industry: The Value of Dealership Visits," Working Paper.

Zhou, J. (2017): "Competitive bundling," Econometrica, 85(1), 145-172. 


\section{A Formal Proof: Search Costs Increase Persistence}

In this section, we show that choice persistence increases in search costs. We define persistence as the probability of a given consumer purchasing the same product in two consecutive search sessions. To illustrate the generality of this result, we prove it for two models most commonly used in the consumer search literature, namely sequential and simultaneous search models. Specifically, we show that

$$
\frac{\partial \operatorname{Pr}\left(y_{t}=y_{t-1}\right)}{\partial c}>0
$$

where $y_{t}$ and $y_{i-1}$ are purchase decisions of a specific consumer in sessions $t$ and $t-1$, and where we suppress consumer index $i$ to simplify notation.

Throughout this section, we assume that the utility for product $j$ in session $t$ is given by

$$
u_{j t}=\xi_{j}+\varepsilon_{j t} .
$$

where $\xi_{j}$ and $\left(\xi_{j}+\varepsilon_{j t}\right)$ denote pre- and post-search utilities. We assume that $\varepsilon_{j t}$ are independent and identically distributed across products and sessions with $\operatorname{cdf} F(\varepsilon)$. Note that we do not take a stance on which product attributes are included in $\xi_{j}$ and $\varepsilon_{j t}$ respectively. The post-search part of of utility $\varepsilon_{j t}$ can be interpreted as a product-specific match value, price, or some other product attribute which is not known before search. We consider the case in which pre-search utilities $\xi_{j}$ are time-invariant. This assumption implies that in both sequential and simultaneous search models, the order in which the consumer adds products to the search set is deterministic and follows descending pre-search utilities $\xi_{j}{ }^{34}$

Since pre-search utilities are time-invariant, and post-search utilities are independent over time, the purchase probability of a given product remains identical across search sessions. Therefore, the probability of choosing the same product in consecutive time periods is given by

$$
\operatorname{Pr}\left(y_{t}=y_{t-1}\right)=\sum_{j} \operatorname{Pr}\left(y_{t}=j\right)^{2}
$$

The general strategy of our proof is the following. We first show that choice persistence always increases when we reduce the purchase share of a given product and relocate that probability mass to other products with higher purchase probabilities. In a second step, we show that increasing search costs induces a series of changes that relocate purchase probabilities as described in the previous result; therefore, any increase in search costs necessarily increases choice persistence.

\footnotetext{
${ }^{34}$ In the sequential search model, assuming that taste shocks $\varepsilon_{j t}$ have identical variances across products implies that products' reservation utilities are ordered in the same way as their pre-search utilities $\xi_{j}$ (Weitzman, 1979). Similarly, in the simultaneous search model, the assumption of identical variances implies that the utility distributions of different products are ordered in the sense of first-order stochastic dominance. The consumer then finds it optimal to expand the search set by adding products in the order of descending mean utilities, which coincides with the order of descending pre-search utilities $\xi_{j}$ (Chade and Smith, 2006).
} 
Relocating purchase probabilities increases persistence We start by showing that choice persistence increases when the purchase share of a specific product $k, s_{k}$, is relocated to products with higher purchase probabilities. We assume products are ordered in terms of descending purchase probabilities: $s_{1}>s_{2}>\ldots>s_{J}$.

Proposition 1. Suppose the purchase share of product $k$, is reduced by $\alpha_{k} \cdot s_{k}$ where $\alpha_{k} \in(0,1]$. At the same time, suppose that the probability mass $\alpha_{k} \cdot s_{k}$ is relocated to products $1, \ldots, k-1$ so that $s_{1}$ increases by $\alpha_{1} \cdot s_{k}, s_{2}$ increases by $\alpha_{2} \cdot s_{k}$, and so on, where $\alpha_{1}, \ldots, \alpha_{k-1}$ are positive allocation weights that sum to $\alpha_{k}$ (i.e., $\alpha_{1} \geq 0, \ldots, \alpha_{k-1} \geq 0$ and $\sum_{j=1}^{j=k-1} \alpha_{j}=\alpha_{k}$ ). If $\operatorname{Pr}\left(y_{t}=y_{t-1}\right)$ and $\tilde{P} r\left(y_{t}=y_{t-1}\right)$ denote choice persistence before and after such relocation, then we always have $\tilde{\operatorname{Pr}}\left(y_{t}=y_{t-1}\right)>\operatorname{Pr}\left(y_{t}=y_{t-1}\right)$.

Proof. We compute choice persistence $\tilde{\operatorname{Pr}}\left(y_{t}=y_{t-1}\right)$ after the relocation of purchase shares by plugging new purchase shares into equation (27):

$$
\begin{aligned}
\tilde{\operatorname{Pr}}\left(y_{t}=y_{t-1}\right) & =\sum_{j=1}^{j=k-1}\left(s_{j}+\alpha_{j} s_{k}\right)^{2}+\left(\left(1-\alpha_{k}\right) s_{k}\right)^{2}+\sum_{j=k+1}^{j=J} s_{j}^{2} \\
& =\sum_{j=1}^{j=k-1}\left(s_{j}^{2}+2 \alpha_{j} s_{j} s_{k}+\left(\alpha_{j} s_{k}\right)^{2}\right)+\left(1-2 \alpha_{k}+\alpha_{k}^{2}\right) s_{k}^{2}+\sum_{j=k+1}^{j=J} s_{j}^{2} \\
& =\operatorname{Pr}\left(y_{t}=y_{t-1}\right)+s_{k}^{2}\left[\sum_{j=1}^{j=k} \alpha_{j}^{2}+2\left(\sum_{j=1}^{j=k-1} \alpha_{j} \frac{s_{j}}{s_{k}}-\alpha_{k}\right)\right]
\end{aligned}
$$

The first term in square brackets is a sum of squared allocation shares that is always non-negative, i.e., $\sum_{j=1}^{j=k} \alpha_{j}^{2} \geq 0$. The second term in square brackets is positive because $s_{j}>s_{k}$ for $\forall j<k$ by assumption, which implies that for those products $\frac{s_{j}}{s_{k}}>1$ and that $\sum_{j=1}^{j=k-1} \alpha_{j} \frac{s_{j}}{s_{k}}-\alpha_{k}>$ $\sum_{j=1}^{j=k-1} \alpha_{j}-\alpha_{k}=0$. It follows that the complete expression in square brackets is positive, implying that $\tilde{\operatorname{Pr}}\left(y_{t}=y_{t-1}\right)>\operatorname{Pr}\left(y_{t}=y_{t-1}\right)$.

Intuitively, Proposition 1 states that when products are ordered in terms of their purchase probabilities, any relocation in purchase probabilities "upwards" (i.e. from any specific product to any combination of products with higher purchase shares) leads to an increase in persistence. For simplicity of exposition, we will refer to this type of change in purchase shares as an "upward relocation".

\section{Sequential Search}

To analyze the sequential search case, we rely on the insight that the sequential search model can be equivalently formulated as a discrete choice problem (Armstrong, 2017; Choi et al., 2018). Specifically, these papers show that the search model can be equivalently represented as a discrete choice model where the consumer chooses a product with the highest effective utility $w_{k t}$, defined as $w_{k t}=\min \left(u_{k t}, z_{k}\right)$ where $u_{k t}$ is the post-search utility of product $k$ and $z_{k}$ is its reservation utility. 
Following Choi et al. (2018), we decompose the reservation utility of product $k$ as $z_{k}=\xi_{k}+\varepsilon_{k}^{*}$, where $\varepsilon_{k}^{*}$ denotes the threshold value for product $k$ that decreases monotonically in search costs. The effective utility of product $k$ can then be expressed as $w_{k t}=\xi_{k}+\min \left(\varepsilon_{k t}, \varepsilon_{k}^{*}\right)$.

Using this expression, we can compute the probability that product $j$ is purchased as

$$
\begin{aligned}
s_{j} & =\operatorname{Pr}\left(w_{j t} \geq w_{k t} \quad \forall k \in J\right) \\
& =\operatorname{Pr}\left(\xi_{j}+\min \left(\varepsilon_{j t}, \varepsilon_{j}^{*}\right) \geq \xi_{k}+\min \left(\varepsilon_{k t}, \varepsilon_{k}^{*}\right) \quad \forall k \in J\right) .
\end{aligned}
$$

Without loss of generality, we let the product index $j \in\{1, \ldots, J\}$ denote the ordering of products in terms of their pre-search utilities, so that $\xi_{1}>\xi_{2}>\ldots>\xi_{J}$ and therefore $z_{1}>z_{2}>\ldots>z_{J}$.

The following proposition shows that increasing the search cost of one specific product generates an upward relocation of purchase shares

Proposition 2. Consider the case where product-specific search costs obey the following restrictions: $c_{k}=c_{j}$ for products $k \leq j$ and $c_{k} \geq c_{j}$ for products $k>j$. Suppose the search cost of product $j, c_{j}$, is increased by some amount $\triangle>0$ small enough such that the order of search remains unaffected. It follows that purchase share $s_{k}$ weakly decreases for product $k=j$, weakly increases for products $k<j$, and remains the same for products $k>j$. Formally, we have

$$
\begin{aligned}
& \text { (1) } s_{k}\left(c_{j}+\triangle\right)-s_{k}\left(c_{j}\right) \leq 0 \text { for } k=j \\
& \text { (2) } s_{k}\left(c_{j}+\triangle\right)-s_{k}\left(c_{j}\right) \geq 0 \text { for } k<j \\
& \text { (3) } s_{k}\left(c_{j}+\triangle\right)-s_{k}\left(c_{j}\right)=0 \text { for } k>j
\end{aligned}
$$

Proof. Since $\varepsilon_{k}^{*}$ is decreasing in search costs, a higher value of the search cost $c_{j}$ implies a lower value of the threshold $\varepsilon_{j}^{*}$ and hence the effective utility of product $j$ weakly decreases

$$
w_{j}\left(c_{j}+\triangle\right)=\xi_{j}+\min \left(\varepsilon_{j t}, \varepsilon_{j}^{*}\left(c_{j}+\triangle\right)\right) \leq \xi_{j}+\min \left(\varepsilon_{j t}, \varepsilon_{j}^{*}\left(c_{j}\right)\right)=w_{j}\left(c_{j}\right)
$$

Because $w_{j}$ is weakly smaller for all realizations of $\varepsilon_{j t}$ and effective utilities for other products remain unchanged, the purchase probability of $j$ weakly decreases and the purchase probability of all other products weakly increases. This proves statements (1) and (2).

Consider products $k>j$. If $\varepsilon_{j}<\varepsilon_{j}^{*}\left(c_{j}+\triangle\right)$, then the effective utility of product $j$ remains the same, that is, $w_{j}\left(c_{j}+\triangle\right)=w_{j}\left(c_{j}\right)$. In this case, the comparison between $w_{j}$ and $w_{k}$ remains unchanged, so $w_{j}\left(c_{j}+\triangle\right) \geq w_{k}$ if and only if $w_{j}\left(c_{j}\right) \geq w_{k}$.

Next, consider the other case where $\varepsilon_{j} \geq \varepsilon_{j}^{*}\left(c_{j}+\triangle\right)$. Since we assume that the order of search is unchanged, the reservation utilities of $j$ and $k$ are still ordered in the same way, so that $z_{j}\left(c_{j}+\triangle\right)>z_{k}$. Using this observation, we can show that the effective utility of product $j$ is 
strictly larger than the effective utility of product $k$ :

$$
\begin{aligned}
w_{j}\left(c_{j}+\triangle\right) & =\xi_{j}+\varepsilon_{j}^{*}\left(c_{j}+\triangle\right) \\
& =z_{j}\left(c_{j}+\triangle\right) \\
& >z_{k} \\
& =\xi_{k}+\varepsilon_{k}^{*} \\
& \geq w_{k}
\end{aligned}
$$

where the first equality follows because $\varepsilon_{j t} \geq \varepsilon_{j}^{*}\left(c_{j}+\triangle\right)$ and hence $\min \left(\varepsilon_{j t}, \varepsilon_{j}^{*}\left(c_{j}+\triangle\right)\right)=\varepsilon_{j}^{*}\left(c_{j}+\right.$ $\triangle)$. The second and forth equality follow from the definition of the reservation utility. The final inequality follows because the highest possible value $w_{k}$ can take on is when the second element in the the minimum function of $w_{k}$ is binding. In summary, we have

$$
w_{j}\left(c_{j}\right) \geq w_{j}\left(c_{j}+\triangle\right)>w_{k}
$$

and hence product $j$ is chosen over product $k$ under search cost $c_{j}$ and under the new search cost $c_{j}+\Delta$.

Therefore, $w_{j}\left(c_{j}+\triangle\right) \geq w_{k}$ if and only if $w_{j}\left(c_{j}\right) \geq w_{k}$ for all realization of $\varepsilon_{j}$. Because the effective utilities for other products remain unchanged, it follows that $s_{k}\left(c_{j}+\triangle\right)-s_{k}\left(c_{j}\right)=0$ for $k>j$.

Intuitively, Proposition 2 states that an increase in product-specific search costs decreases the purchase probability for the focal product, increases purchase probabilities for any product searched earlier, and does not affect purchase probabilities of products searched later. Therefore, this change in purchase shares constitutes an upward relocation. By combining Propositions 1 and 2, we can now show that in the sequential search model persistence increases when search costs increase.

Theorem 1. In the sequential search model, increasing search costs of all products by a fixed amount $\Delta>0$ leads to an increase in choice persistence for any value of $\Delta$.

Proof. To prove this theorem, we first note that for a given level of search costs, products with higher pre-search utilities $\xi_{j}$ also have higher purchase probabilities. This follows because $s_{j}=$ $\operatorname{Pr}\left(\xi_{j}+\min \left(\varepsilon_{j t}, \varepsilon_{j}^{*}\right) \geq \xi_{k}+\min \left(\varepsilon_{k t}, \varepsilon_{k}^{*}\right) \quad \forall k \in J\right)$, where in the case of uniform search costs and iid taste shocks $\varepsilon_{j t}$, the term $\min \left(\varepsilon_{j t}, \varepsilon_{j}^{*}\right)$ is identically distributed across products. Therefore, a higher value of $\xi_{j}$ leads to a higher purchase share $s_{j}$.

We now construct a series of product-specific changes in search costs that generate upward relocations of purchase shares. We start from a given search cost level $c_{1}$ which is equal across products and then increase search costs by a given amount for each product such that new search costs are equal to $c_{1}+\Delta$. We first increase search costs in this fashion for the lowest reservation utility product and continue in ascending order of reservation utilities. According to Proposition 2, increasing search costs for the lowest reservation utility product $J$ leads to an upward relocation of 
purchase shares from product $J$, which has the lowest purchase share, to all other products. The search cost increase does not change search order, because product $J$ has the lowest reservation utility before the change in search costs and the increase in search costs further lowers its reservation utility.

Next, we increase search costs by the same amount for product $J-1$. Using Proposition 2 again, we conclude that this change in search costs leads an upward relocation of purchase shares from product $J-1$ to all other products $j<J-1$. Search order does not change because after the change in search costs for $J-1$ all products $j<J-1$ have the identical search costs, while all products $j \geq J-1$ have identical search costs but higher than that of products $j<J-1$. Therefore, the order of reservation utilities does not change. We continue this process until we reach product $j=1$. When search costs are altered for the final product, purchase shares do not change, because $j=1$ continues to be searched first.

Once the sequence of relocations is completed, the search costs of all products have increased to $c_{2}=c_{1}+\Delta$. Because each individual change in the constructed sequence is an upward relocation, it follows from Proposition 1 that choice persistence has increased.

\section{Simultaneous Search}

To prove our result for the simultaneous search model, we rely on several properties of the optimal search strategy. We state these properties without proofs, but an interested reader may consult Chade and Smith (2006) for the relevant derivations. As before, we assume that products are ordered by their pre-search utilities $\xi_{j}$.

1. The consumer adds products to the search set in order of decreasing pre-search utilities $\xi_{j}$.

2. For a given level of search costs, the consumer's search set size is deterministic. Search set size does not depend on the realizations of post-search utilities $\left(\xi_{j}+\varepsilon_{j t}\right)$ because the consumer commits to a search set size before starting search.

3. Search set size is weakly decreasing in search costs.

4. Purchase shares are ordered in the same way as pre-search utilities, so purchase probabilities decrease in $j$.

In the following proposition, we show that increasing the search cost in the simultaneous search model leads to a change in purchase probabilities from the products that are no longer searched to the products with the highest pre-search utilities.

Proposition 3. Suppose search costs of all products are increased by some amount $\triangle>0$ from $c_{1}$ to $c_{2}=c_{1}+\triangle$. If the consumer optimally searched $k_{1}$ products before the change, then there exists a product $k_{2} \leq k_{1}$ such that purchase shares $s_{j}$ weakly increase for products $j \leq k_{2}$ and weakly decrease for products $j>k_{2}$. Formally, we have 


$$
\begin{aligned}
& \text { (1) } s_{j}\left(c_{1}+\triangle\right)-s_{j}\left(c_{1}\right) \geq 0 \text { for } j \leq k_{2} \\
& \text { (2) } s_{j}\left(c_{1}+\triangle\right)-s_{j}\left(c_{1}\right) \leq 0 \text { for } j>k_{2}
\end{aligned}
$$

Proof. If the optimal search set size is $k_{1}$ products under the search $\operatorname{cost} c_{1}$, then purchase probabilities are given by

$$
\begin{array}{rlrl}
\operatorname{Pr}_{j}\left(c_{1}\right)=\operatorname{Pr}\left(\xi_{j}+\varepsilon_{j t} \geq \xi_{l}+\varepsilon_{l t}\right. & \left.\forall l \in\left\{1, \ldots, k_{1}\right\}\right) & & \text { if } j \leq k_{1} \\
=0 & & \text { if } j>k_{1}
\end{array}
$$

Any product $j>k_{1}$ not included in the search set has a purchase probability of zero. When search costs increase from $c_{1}$ to $c_{2}$, the search set size weakly decreases and hence purchase probabilities are now given by

$$
\begin{array}{rrr}
\operatorname{Pr}_{j}\left(c_{2}\right)=\operatorname{Pr}\left(\xi_{j}+\varepsilon_{j t} \geq \xi_{l}+\varepsilon_{l t}\right. & \left.\forall l \in\left\{1, \ldots, k_{2}\right\}\right) & \text { if } j \leq k_{2} \\
=0 & \text { if } j>k_{2}
\end{array}
$$

where $k_{2} \leq k_{1}$.

Comparing the two sets of purchase shares, we conclude that if search set size remains the same, purchase probabilities also stay the same. If the search set size decreases such that $k_{2}<k_{1}$ with strict inequality, then the purchase probabilities for products $k_{2}<j \leq k_{1}$ decrease to zero and remain zero for any product $j>k_{1}$ that was not searched at the previous level of search costs. At the same time, the purchase probabilities of products $j \leq k_{2}$ increase. To see this, note that

$$
\begin{aligned}
\operatorname{Pr}\left(\xi_{j}+\varepsilon_{j t} \geq \xi_{l}+\varepsilon_{l t} \quad \forall l \in\left\{1, \ldots, k_{1}\right\}\right)= & \operatorname{Pr}\left(\xi_{j}+\varepsilon_{j t} \geq \xi_{l}+\varepsilon_{l t} \quad \forall l \in\left\{1, \ldots, k_{2}\right\}\right. \\
& \text { AND } \left.\quad \xi_{j}+\varepsilon_{j t} \geq \xi_{m}+\varepsilon_{m t} \quad \forall m \in\left\{k_{2}+1, \ldots, k_{1}\right\}\right) \\
< & \operatorname{Pr}\left(\xi_{j}+\varepsilon_{j t} \geq \xi_{l}+\varepsilon_{l t} \quad \forall l \in\left\{1, \ldots, k_{2}\right\}\right)
\end{aligned}
$$

which holds for all products $j$ such that $j \leq k_{2}$. This last inequality holds because, other things equal, each product $j$ is more likely to be selected when it is being chosen from a smaller set.

By combining Propositions 1 and 3, we can now show that in the simultaneous search model persistence increases when search costs increase.

Theorem 2. In the simultaneous search model, increasing search costs of all products by a fixed amount $\Delta \geq 0$ leads to an increase in choice persistence for any value of $\Delta$.

Proof. First, note that products with higher pre-search utility $\xi_{j}$ also have a higher purchase share. ${ }^{35}$

\footnotetext{
${ }^{35}$ This follows from the purchase share expression $\operatorname{Pr}_{j}\left(c_{1}\right)=\operatorname{Pr}\left(\xi_{j}+\varepsilon_{j t} \geq \xi_{l}+\varepsilon_{l t} \quad \forall l \in\left\{1, \ldots, k_{1}\right\}\right)$ for $j \leq k_{1}$
} 

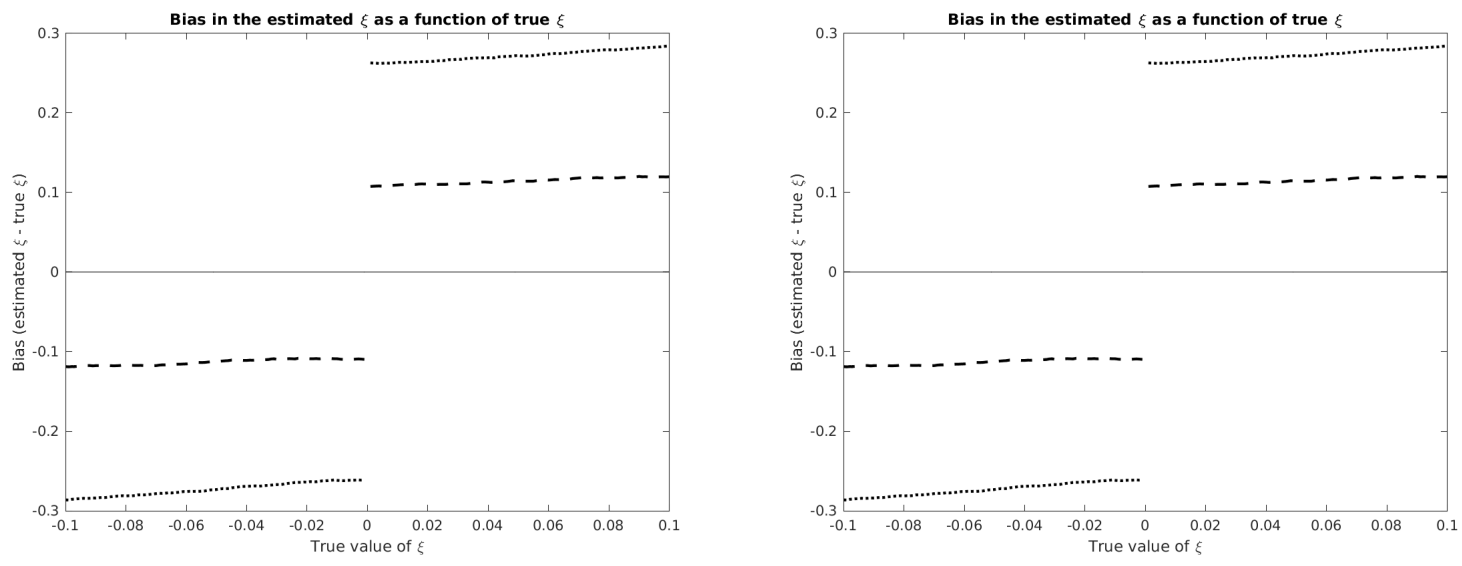

Figure A1: Bias in Inferred Intercepts: 2 Products. The two graphs display the difference between inferred and true intercepts under the assumption of a normal (left graph) or extreme value (right graph) taste shocks. We assume $\xi_{A}=0$ and plot the bias in $\xi_{B}$ as a function of the true intercept value. The solid / dashed / dotted lines correspond to the cases search costs are equal to $0 / 0.25 / 0.4$.

Let $c_{1}$ denote the initial level of search costs, and let $c_{2}=c_{1}+\Delta$ denote its value after the change. According to Proposition 3, there exists a product $k_{2} \leq k_{1}$ such that when search costs change from $c_{1}$ to $c_{2}$, purchase shares $s_{j}$ weakly increase for products $j \leq k_{2}$ and weakly decrease for products $j>k_{2}$. Starting from the initial purchase probabilities under $c_{1}$, we can iteratively decrease the purchase probability of the product with the lowest non-zero purchase share for all products $k_{2} \leq j<k_{1}$, each time relocating the probability mass to products $j \leq k_{2}$. Each of these relocations lead to a new distribution of purchase shares in which shares of products $j \leq k_{2}$ become weakly higher. Hence, increasing search costs from $c_{1}$ to $c_{2}$ induces changes in purchase shares that can be achieved by a series of upward relocations of this kind. It then follows from Proposition 1 that choice persistence increases.

\section{B Search Costs Increase Estimates of Preference Heterogeneity}

In this section we show that in the presence of positive search costs, the bias in the inferred intercept of a given product $\left(\hat{\xi}_{j}-\xi_{j}\right)$ is increasing in $\xi_{j}$. From this statement it follows that for any pair of consumers, denoted as consumer 1 and 2, it holds that inferred intercepts are further apart from each other than true intercepts. For example, when $\xi_{1 j}>\xi_{2 j}$, then we infer intercepts are such that $\hat{\xi}_{1 j}-\hat{\xi}_{2 j}>\xi_{1 j}-\xi_{2 j}$ and therefore search costs increase the dispersion in inferred intercepts across consumers. Furthermore, the absolute value of the bias is increasing in search costs, thus leading to a stronger increase in dispersion if search costs are higher.

We show that this result holds through an extensive set of simulations. We first simulate consumer behavior for a market with two products, where $\xi_{A}$ is normalized to zero, and where we and $\operatorname{Pr}_{j}\left(c_{1}\right)=0$ for $j>k_{1}$. 

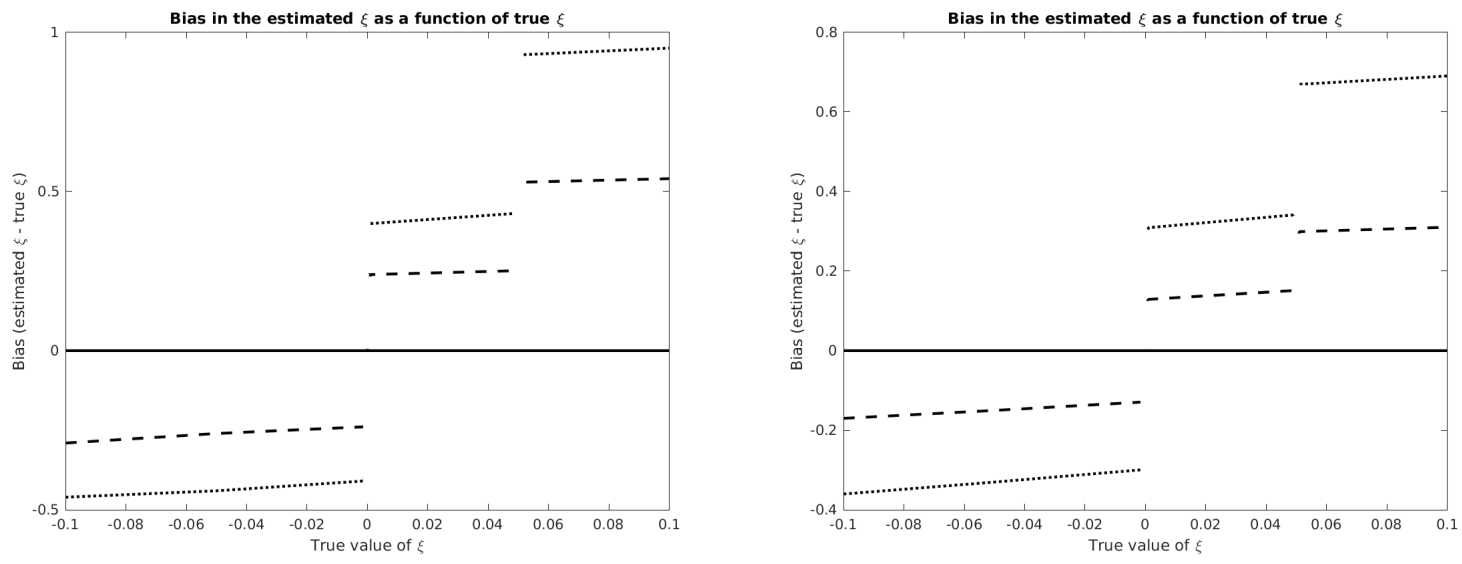

Figure A2: Bias in Inferred Intercepts: 3 Products. The two graphs display the difference between inferred and true intercepts under the assumption of a normal (left graph) or extreme value (right graph) taste shocks. We assume $\xi_{A}=0, \xi_{B}=0.05$ and plot the bias in $\xi_{C}$ as a function of the true intercept value. The solid / dashed / dotted lines correspond to the cases search costs are equal to $0 / 0.25 / 0.4$.

change the true value of $\xi_{B}$. Figure A1 plots the bias in the inferred intercept as a function of the true intercept value (for the one non-normalized product). We repeat this exercise for two distributions of taste shocks that are commonly used in discrete choice demand models: normal (left graph) and extreme value (right graph). The graphs show that the sign of the bias in inferred intercepts coincides with the sign of the true intercept $\xi_{B}$, so that positive intercepts are overestimated and negative intercepts are underestimated. ${ }^{36}$ Moreover, the absolute value of the bias $\left|\hat{\xi}_{B}-\xi_{B}\right|$ increases in the absolute value of the intercept $\left|\xi_{B}\right|$. Therefore, the bias is increasing in the value of the true intercept and for any pair of consumers, the inferred intercepts $\hat{\xi}_{1 B}$ and $\hat{\xi}_{2 B}$ are further apart from each other than true intercepts $\xi_{1 B}$ and $\xi_{2 B}$. Furthermore, the absolute value of the bias increases in search costs and therefore higher search costs lead to higher inferred heterogeneity in preferences.

We repeat these simulations for a case with three products where we fix the intercepts of two products and compute the bias in the intercept of the third product for different intercept values. The two graphs in Figure A2 show the results from such a simulation for the case where we set $\xi_{A}=0$ and $\xi_{B}=0.05$ and vary the intercept of the third product, $\xi_{C}$. Similar to the two-product case, we find that intercepts are overestimated (underestimated) if the true intercept is positive (negative) and the bias is increasing in the value of the true intercept. Furthermore, the absolute value of the bias is increasing in search costs.

We repeat similar simulation exercises for other distributions of taste shocks $\varepsilon$ (logistic and uniform), as well as for settings with more than 3 products, and find that throughout all simulations inferred heterogeneity in preferences is increasing in search costs.

\footnotetext{
${ }^{36} \mathrm{~A}$ discontinuity in the bias occurs at $\xi_{B}=0$ because as $\xi_{B}$ switches from negative to positive, the consumer changes the search order to searching product $B$ first.
} 


\section{Normalization of Taste Shock Variances}

When estimating our model, we normalize the variances of both taste shocks to one, setting $\sigma_{\mu}^{2}=1$ and $\sigma_{\varepsilon}^{2}=1$. In this appendix, we discuss why we need both normalizations and explain why they prevent us from monetizing the estimates of search costs.

To illustrate the role of two normalizations, we start by writing consumer utility in its nonnormalized form:

$$
u_{i j t}=\xi_{i j}-\alpha_{i} p_{i j t}+\sigma_{\mu} \mu_{i j t}+\sigma_{\varepsilon} \varepsilon_{i j t}
$$

where taste shocks $\mu_{i j t}$ and $\varepsilon_{i j t}$ are i.i.d. and follow the standard normal distribution. Next, we divide both utility and search costs by the standard deviation $\sigma_{\mu}$ of the pre-search shocks to obtain:

$$
\begin{aligned}
\tilde{u}_{i j t} & =\xi_{i j} / \sigma_{\mu}-\left(\alpha_{i} / \sigma_{\mu}\right) p_{i j t}+\mu_{i j t}+\left(\sigma_{\varepsilon} / \sigma_{\mu}\right) \varepsilon_{i j t} \\
& =\tilde{\xi}_{i j}-\tilde{\alpha}_{i} p_{i j t}+\mu_{i j t}+\tilde{\sigma}_{\varepsilon} \varepsilon_{i j t} \\
\tilde{c}_{i j} & =c_{i j} / \sigma_{\mu}
\end{aligned}
$$

where we use the tilde notation for all variables scaled by $\sigma_{\mu}$ (e.g. $\left.\tilde{u}_{i j t}=u_{i j t} / \sigma_{\mu}\right)$. Since this transformation rescales utilities, search costs, and reservation utilities in the same way, we have not altered search order, stopping, or purchase decisions of consumers. Thus, we set $\sigma_{\mu}=1$ without loss of generality.

Now consider estimating the standard deviation of post-search shocks, $\tilde{\sigma}_{\varepsilon}$. Since increasing this standard deviation $\tilde{\sigma}_{\varepsilon}$ and decreasing search costs both lead to more search, we can only identify search costs relative to the post-search shock variance $\tilde{\sigma}_{\varepsilon}$. Although search costs and $\tilde{\sigma}_{\varepsilon}$ are not fully colinear, in practice multiplying the post-search standard deviation by a factor $k$ roughly scales the estimated search costs by a factor of $k$, while leaving other parameters mostly unchanged. Therefore, there appears to be little variation in the data that would help us estimate $\tilde{\sigma}_{\varepsilon}$, which is why we set it equal to one in our application.

These normalizations have one important implication for how we interpret estimated parameters. While we can monetize our estimates of product intercepts, we are unable to monetize search costs. As discussed above, we can only identify search costs relative to the standard deviation $\tilde{\sigma}_{\varepsilon}$; therefore, the estimated search cost in the re-scaled model is given by:

$$
\frac{\tilde{c}_{i j}}{\tilde{\sigma}_{\varepsilon}}=\frac{c_{i j} / \sigma_{\mu}}{\sigma_{\varepsilon} / \sigma_{\mu}}=c_{i j} / \sigma_{\varepsilon}
$$

Therefore, when we calculate the ratio of the estimated search costs to the estimated price coefficient, we are computing the ratio of $\alpha_{i} / \sigma_{\mu}$ and $c_{i j} / \sigma_{\varepsilon}$, which does not equal to the monetary value of search costs. However, $\sigma_{\varepsilon}$ affects only estimated search costs but not utility parameters known to the consumer prior to searching, such as the price coefficient and product intercepts. Therefore, we cannot monetize search costs but can monetize product intercepts. 

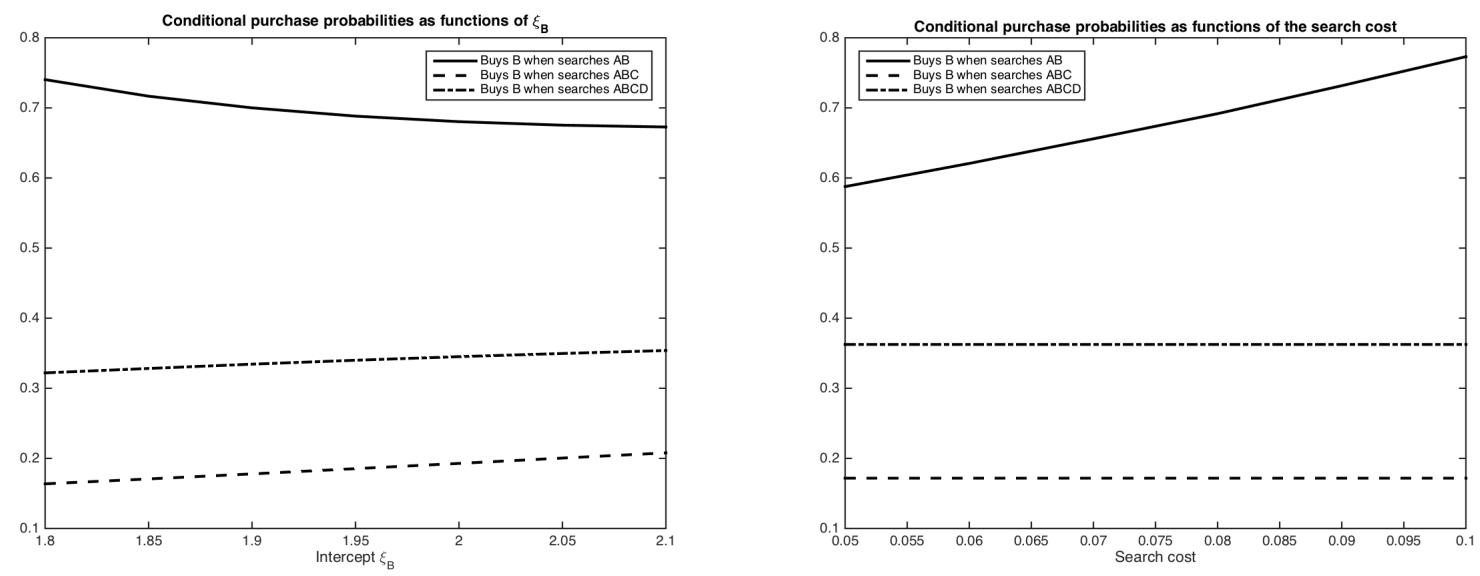

Figure A3: Conditional Purchase Probabilities when Varying Intercept Value or Search Costs.

\section{Identification: The Role of Purchase Data}

In this section, we illustrate how conditional purchase probabilities behave as a function of product intercepts and product-specific search costs. To simplify exposition, we consider a setting with 4 products - A, B, C, D - and focus on the behavior of a consumer that searches these products in alphabetic order. We first set $\xi_{A}=2.2, \xi_{B}=1.8, \xi_{C}=1.6, \xi_{D}=1.4$ and $c_{A}=c_{B}=c_{C}=c_{D}=0.1$. For simplicity, we omit the pre-search taste shock in this example. Starting from this baseline, we analyze changes in behavior in response to two changes in model parameters. First, we increase the intercept of product B, and second, we decrease product-specific search cost for product B. In both cases, we analyze changes that are small enough so that search order remains constant. In Figure A3, we plot the purchase probability for product B conditional on different sets of searched products when altering either the intercept value or search costs. The graphs show that the change in the intercept leads to a higher purchase probabilities for product $\mathrm{B}$ conditional on searching products $\mathrm{A}, \mathrm{B}$, and $\mathrm{C}$ or $\mathrm{A}, \mathrm{B}, \mathrm{C}$, and $\mathrm{D}$ (i.e. sets where product $\mathrm{B}$ is not the last product the consumer searched), whereas the change in the search cost leaves those probabilities unchanged. Therefore, product-specific rates of recall (the probability of purchasing a product searched earlier in the search sequence) can help us identify preferences separately from search costs.

The intuition for the difference in behavior highlighted in Figure A3 can be gleaned from equations (6) and (7). The probability of continuing or stopping does not depend on the reservation utilities of products searched previously but does depend on realized utilities of those products. Therefore, the distribution of post-search taste shocks conditional on searching a specific set of products does not change when altering search costs of any product that is not the last product searched. As a consequence, conditional purchase probabilities remain the same. Instead, increasing the intercept does change the continuation and stopping decisions at each step, thus leading to a different conditional distribution of post-search taste shocks in different searched sets and therefore to different conditional purchase probabilities. 
We also note that it is not generally true that conditional purchase probabilities are unaffected by changes in search costs. In the example above, the purchase probability of product $\mathrm{B}$ conditional on searching $\mathrm{A}$ and $\mathrm{B}$ does change when altering the search cost of product $\mathrm{B}$ (see the right-hand side graph in Figure A3). This occurs because the realizations of post-search taste shocks for which set $(A, B)$ is optimal are a function of product B's reservation utility (because product B is the last product searched) and hence change when this product's search costs are altered. In this particular example, lowering the search cost of product B makes it more likely that the consumer searches product B (i.e. continues to search after evaluating product A), even if the post-search taste shock draw for product $\mathrm{A}$ is relatively favorable, and hence the purchase probability of product B conditional on searching A and B decreases.

\section{E Estimation Details}

In section 5, we outlined the main features of the importance-sampling estimator; this section provides additional details. With regards to simulation draws, we set $N_{S}=1000$ and $N_{M}=100$. That is, for each consumer, we take 100 draws from the proposal distribution of types $g\left(\theta_{i}\right)$. For each of these draws, we take 1000 draws from the distributions of utility shocks $\varepsilon_{i t}$ and $\mu_{i t}$ for each consumer-session pair.

To compute individual likelihoods in (23), we need to first calculate reservation utilities $z_{i j t}$ for each set of random draws $\theta_{i}$ and $\mu_{i j t}$. Calculating reservation utilities is computationally burdensome, so in practice we use approximations. Recall that $z_{i j t}$ is defined from the following indifference condition, which is equivalent to the equation (2):

$$
z_{i j t}=-c_{i j}+P\left(u_{i j t} \geq z_{i j t}\right) E\left(u_{i j t} \mid u_{i j t} \geq z_{i j t}\right)+P\left(u_{i j t}<z_{i j t}\right) z_{i j t} .
$$

Under our assumption that $\varepsilon_{i j t}$ follows a normal distribution with standard deviation $\sigma_{\varepsilon}$, this condition can be written in a more convenient form:

$$
z_{i j t}=\left(z_{i j t}-\delta_{i j t}\right) \Phi\left(\frac{z_{i j t}-\delta_{i j t}}{\sigma_{\varepsilon}}\right)+\sigma_{\varepsilon} \cdot \phi\left(\frac{z_{i j t}-\delta_{i j t}}{\sigma_{\varepsilon}}\right)+\delta_{i j t}-c_{i j},
$$

where $\Phi(\cdot)$ and $\phi(\cdot)$ represent the Gaussian CDF and PDF. The right-hand side of this expression is a contraction mapping with respect to $z_{i j t}$, so we can compute reservation utilities through a fixedpoint iteration procedure (see Elberg, Gardete, Macera, and Noton, 2017). To ease computation, we precompute a lookup table that stores the values of reservation utilities $z_{i j t}$ for a large number of combinations of $\delta_{i j t}$ and $c_{i j}$. We compute relevant reservation utilities from this lookup table using linear interpolation. This method is computationally inexpensive, because we only need to compute the lookup table once before the estimation. 


\section{F Additional Details: Persistence in Information}

In this section we provide additional details on the extension of our main model that allows for correlation in post-search taste shocks across search sessions. Recall that in this extended model, taste shocks $\varepsilon_{i j t}$ follow an MA(1) process so that $\varepsilon_{i j t}=\omega_{i j t}+\rho \omega_{i j t-1}$ where $\omega_{i j t}$ is drawn from $N(0,1)$ and is iid across consumers, products, and search sessions. Upon searching product $j$ in period $t$, consumer $i$ observes both the value of the post-search shock $\varepsilon_{i j t}$ and the value of the transitory shock $\omega_{i j t}$. These assumptions imply the following information structure. If consumer $i$ did not search product $j$ in period $t-1$, she expects $\varepsilon_{i j t}$ to be distributed according to $\varepsilon_{i j t} \sim$ $N\left(0, \sigma_{\omega}^{2} \cdot\left(1+\rho^{2}\right)\right)$. By contrast, if consumer $i$ searched product $j$ in the previous period, she expects $\varepsilon_{i j t}$ to be distributed according to $\varepsilon_{i j t} \mid \omega_{i j t-1} \sim N\left(\rho \omega_{i j t-1}, \sigma_{\omega}^{2}\right)$.

The likelihood now also depends on the persistence parameter $\rho$. As before, $D_{i}=\left\{\pi_{i t}, S_{i t}, y_{i t}\right\}_{t=1}^{T_{i}}$ is the data for all $T_{i}$ sessions of consumer $i, D=\left\{D_{i}\right\}_{i=1}^{N}$ is

for all consumers, $\Omega$ is a vector of hyperparameters that determine the distribution of preferences and search costs. We take $N_{M}$ draws of types from the proposal density $g\left(\theta_{i}\right)$ and compute the simulated likelihood:

$$
\tilde{L}(D \mid \Omega, \rho, p)=\prod_{i=1}^{N} \frac{1}{N_{M}} \sum_{m=1}^{N_{M}}\left(\prod_{t=1}^{T_{i}} \tilde{L}_{i t}\left(D_{i t} \mid \theta_{i}^{m}, \rho, p_{i t}\right) \cdot \frac{f\left(\theta_{i}^{m} \mid \Omega\right)}{g\left(\theta_{i}^{m}\right)}\right),
$$

where $\theta_{i}^{m}$ denotes the $m$-th draw of types for consumer $i$, and $\tilde{L}_{i t}\left(D_{i t} \mid \theta_{i}^{m}, \rho, p_{i t}\right)$ is the simulated consumer and session-specific contribution to the likelihood:

$$
\tilde{L}_{i t}\left(D_{i t} \mid \theta_{i}^{m}, \rho, p_{i t}\right)=\frac{1}{N_{S}} \sum_{s=1}^{N_{S}} 1\left\{W_{i t}\left(\theta_{i}^{m}, \mu_{i t}^{s}, \omega_{i t}^{s}, \omega_{i t-1}^{s}, S_{i t-1}^{(s)}, p_{i t}\right) \geq 0\right\} .
$$

In this expression, $\mu_{i t}^{s}, \omega_{i t}^{s}$ and $\omega_{i t-1}^{s}$ are draws of taste shocks and $S_{i t-1}$ is the search set in session $(t-1)$. For $(t>1)$, we observe $S_{i t-1}$ and can simply condition on the search set of the previous period. In the case of $(t=1)$, the previous search set $S_{i 0}$ is unobserved and hence we simulate realizations of $S_{i 0}^{s}$.

To simulate draws $S_{i 0}^{s}$, we simulate decisions of consumer $i$ (for a given type draw $\theta_{i}^{m}$ and persistence parameter $\rho$ ) for a large number of search sessions, initiating simulation at an arbitrary starting point. Throughout this simulation, we set product prices equal to their average levels observed in our dataset. We then discard 1,000 first simulations and consider the remaining simulated sessions to be draws from the stationary distribution $F\left(S_{i 0} \mid \theta_{i}, \rho\right)$.

To estimate the model, we implement a grid search over different values of $\rho$ between -1 and 1. For every candidate value of $\rho$, we simulate $F\left(S_{i 0} \mid \theta_{i}, \rho\right)$ and then estimate the hyperparameters of the preferences and search cost distributions $\Omega$ using our importance sampling method. Our estimation procedure for this extended model then proceeds in the following steps:

1. Fix the correlation parameter $\rho$. 
2. Fix the vector of hyperparameters $\Omega$.

3. Generate draws of consumer types $\theta_{i}$ from the proposal distribution $g\left(\theta_{i}\right)$.

4. For each type draw $\theta_{i}^{m}$, simulate draws $S_{i 0}^{s}$ as explained above.

5. For each type draw $\theta_{i}^{m}$ and each draw of the initial condition $S_{i 0}^{s}$, compute the likelihood $\tilde{L}_{i t}\left(D_{i t} \mid \theta_{i}^{m}, \rho, p_{i t}\right)$ using simulation as in the main model. To simplify estimation, we only use one draw of the initial condition $S_{i 0}^{s}$ but 100 draws for transitory shocks $\omega_{i j t}$ and pre-search shocks $\mu_{i j t}$.

6. Plug the values of the simulated likelihoods $\tilde{L}_{i t}\left(D_{i t} \mid \theta_{i}^{m}, \rho, p_{i t}\right)$ into the importance sampling formula. Average across proposal types $\theta_{i}$ to compute the integrated individual likelihood and multiply likelihoods of all consumers to obtain total likelihood $\tilde{L}(D \mid \Omega, \rho, p)$.

7. Maximize $\tilde{L}(D \mid \Omega, \rho, p)$ with respect to hyperparameters $\Omega$ to obtain an estimate $\hat{\Omega}(\rho)$ conditional on the value of the correlation parameter $\rho$.

8. Repeat steps 4-7 for different candidate values of the correlation parameter $\rho$.

9. Using grid search, maximize $\tilde{L}(D \mid \hat{\Omega}(\rho), \rho, p)$ with respect to the correlation parameter $\rho$.

\section{G Importance Sampling versus Kernel-smoothed Frequency Esti- mator}

In this section we compare our approach with a kernel-smoothed frequency estimator. As we show below, using a kernel-smoothed frequency estimator in our application is computationally more burdensome and relatively slow. To illustrate this point, we generate a simulated dataset that mimics our actual data, and use this simulated dataset to show how the computational burden increases with sample size. Specifically, we estimate a model with 10 products and parametrize the utility function and search cost parameters as in our main specification. For simplicity, we omit the price coefficient and assume that consumers have identical search costs.

Tables A1 and A2 show estimation results obtained using two estimation methods for different sample sizes (50, 100, and 500 consumers). Table A3 summarizes the relative performance of both estimators in terms of CPU time. We find that across all samples sizes, the importance sampling procedure is substantially faster. When extrapolating to a sample size of 4,000 consumers, we conclude that it would take us about 3 days to estimate the model using a kernel-smoothed frequency estimator. By contrast the importance sampling approach allows us to estimated the model in about 3 hours. Additionally, the estimates in Tables A1 and A2 suggest that the importance sampling approach recovers structural parameters somewhat more accurately. As a whole, our results suggest that importance sampling provides a fast and practical way to estimate our model, at the same time retaining similar precision to that of alternative methods. 


\section{H Computation of Personalized Prices}

We compute expected profits as

$$
\Pi\left(p_{j}, H\right)=\sum_{k \in \mathcal{F}} \int\left(p_{k}-m c_{k}\right) \operatorname{Pr}(y=k \mid \theta) f\left(\theta \mid H, p^{H}, \Omega\right) g(\Omega) d \theta d \Omega .
$$

This profit function captures uncertainty regarding both type $\theta$ and structural parameters $\Omega$. To compute personalized prices, we first need to calculate the integrals in (30). These integrals do not have closed-form solutions and have to be approximated using simulation. We take bootstrap estimates $\hat{\Omega}^{b}$ and treat them as draws from the distribution $g(\Omega){ }^{37}$ For each of these draws $\hat{\Omega}^{b}$, we use the Metropolis-Hastings algorithm to take $S$ draws of types $\theta_{s}^{b}$ from the posterior distribution $f\left(\theta \mid H, p^{H}, \hat{\Omega}^{b}\right)$. Next, we use the resulting draws of types and compute the probability that the consumer buys product $j$ for each of these draws. The expected profits in (30) can then be approximated with the following expression:

$$
\Pi\left(p_{j}, H\right) \approx \frac{1}{B \times S} \sum_{k \in \mathcal{F}} \sum_{b=1}^{B} \sum_{s=1}^{S}\left(p_{k}-m c_{k}\right) \operatorname{Pr}\left(y=k \mid \theta_{s}^{b}\right)
$$

Note that $B$ is the number of bootstrap estimates, and $S$ is the number of types we draw for each of these bootstrap estimates.

\footnotetext{
${ }^{37}$ We use the same bootstrap estimates that we obtained earlier to compute standard errors of parameter estimates.
} 


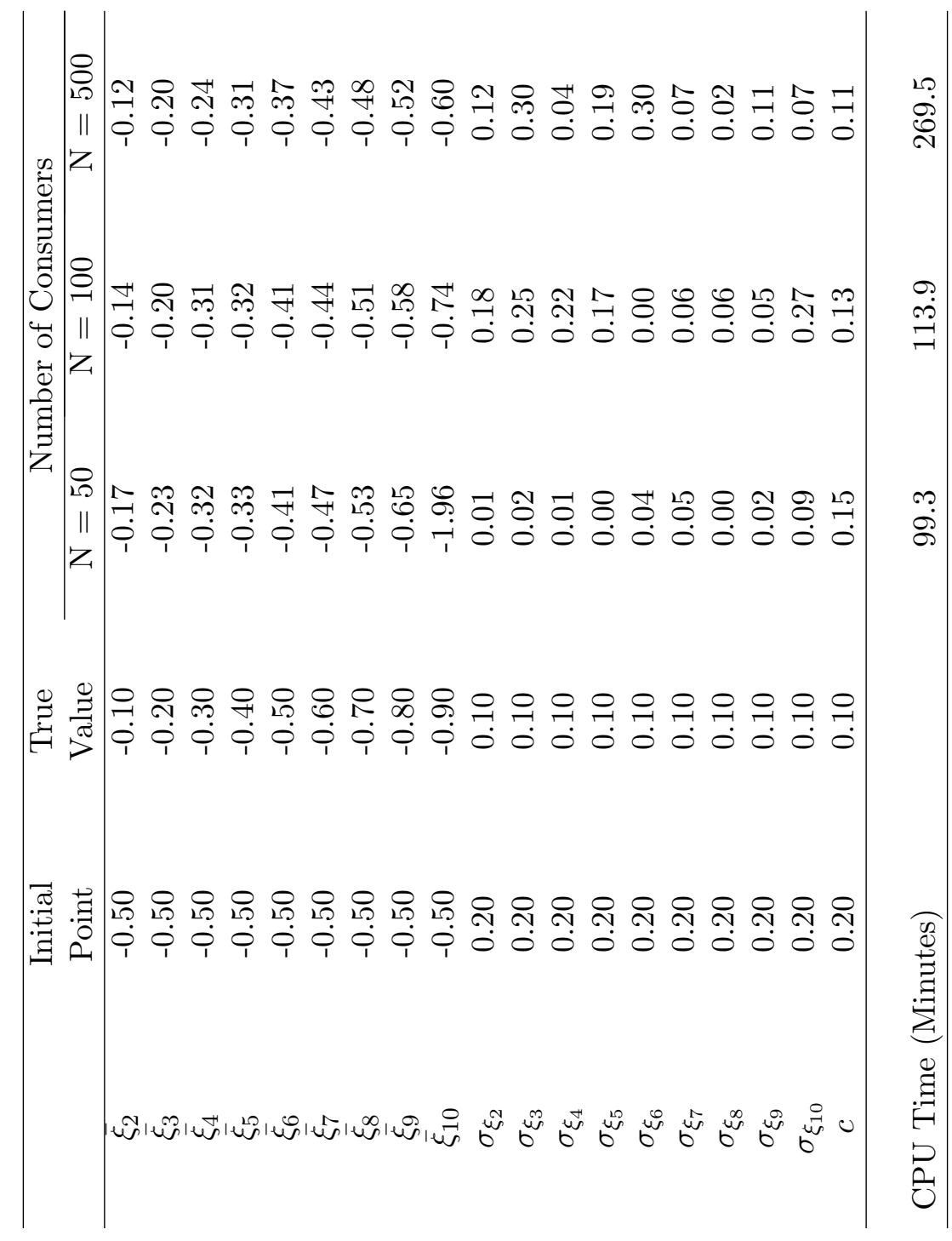

Table A1: Kernel-smoothed Frequency Estimator. The smoothing parameter is fixed at $\mathrm{h}=5$ as in Honka and Chintagunta (2017). The final three columns show the performance of the estimator across three samples of different size. 


\begin{tabular}{|c|c|c|c|c|c|}
\hline & \multirow{2}{*}{$\begin{array}{l}\text { Initial } \\
\text { Point }\end{array}$} & \multirow[b]{2}{*}{ Truth } & \multicolumn{3}{|c|}{ Number of Consumers } \\
\hline & & & $\mathrm{N}=50$ & $\mathrm{~N}=100$ & $\mathrm{~N}=500$ \\
\hline $\bar{\xi}_{2}$ & -0.50 & -0.10 & -0.11 & -0.11 & -0.10 \\
\hline $\bar{\xi}_{3}$ & -0.50 & -0.20 & -0.20 & -0.20 & -0.20 \\
\hline $\bar{\xi}_{4}$ & -0.50 & -0.30 & -0.31 & -0.29 & -0.29 \\
\hline $\bar{\xi}_{5}$ & -0.50 & -0.40 & -0.40 & -0.40 & -0.39 \\
\hline $\bar{\xi}_{6}$ & -0.50 & -0.50 & -0.48 & -0.49 & -0.51 \\
\hline $\bar{\xi}_{7}$ & -0.50 & -0.60 & -0.58 & -0.61 & -0.60 \\
\hline $\bar{\xi}_{8}$ & -0.50 & -0.70 & -0.70 & -0.71 & -0.70 \\
\hline $\bar{\xi}_{9}$ & -0.50 & -0.80 & -0.81 & -0.79 & -0.80 \\
\hline $\bar{\xi}_{10}$ & -0.50 & -0.90 & -0.89 & -0.90 & -0.91 \\
\hline$\sigma_{\xi_{2}}$ & 0.20 & 0.10 & 0.09 & 0.10 & 0.10 \\
\hline$\sigma_{\xi_{3}}$ & 0.20 & 0.10 & 0.10 & 0.09 & 0.10 \\
\hline$\sigma_{\xi_{4}}$ & 0.20 & 0.10 & 0.10 & 0.09 & 0.11 \\
\hline$\sigma_{\xi_{5}}$ & 0.20 & 0.10 & 0.10 & 0.10 & 0.10 \\
\hline$\sigma_{\xi_{6}}$ & 0.20 & 0.10 & 0.10 & 0.12 & 0.10 \\
\hline$\sigma_{\xi_{7}}$ & 0.20 & 0.10 & 0.07 & 0.09 & 0.10 \\
\hline$\sigma_{\xi_{8}}$ & 0.20 & 0.10 & 0.09 & 0.10 & 0.10 \\
\hline$\sigma_{\xi_{9}}$ & 0.20 & 0.10 & 0.11 & 0.14 & 0.13 \\
\hline$\sigma_{\xi_{10}}$ & 0.20 & 0.10 & 0.13 & 0.10 & 0.10 \\
\hline$c$ & 0.20 & 0.10 & 0.10 & 0.10 & 0.10 \\
\hline $\mathrm{Ti}$ & es) & & 8.0 & 19.6 & 33.6 \\
\hline
\end{tabular}

Table A2: Importance Sampling Estimator. The final three columns show the performance of the estimator across three samples of different size. 


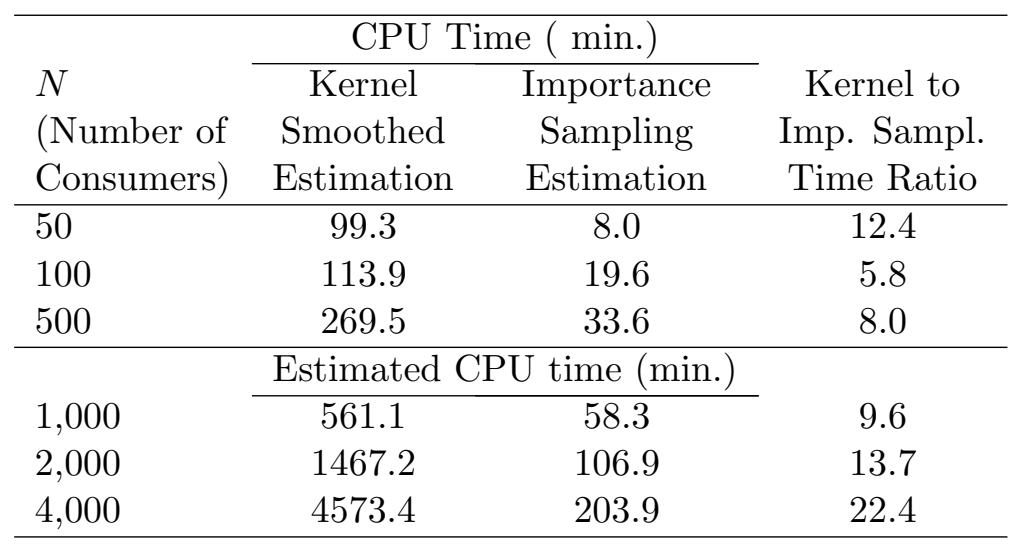

Table A3: CPU Time Comparison: Kernel-smoothed Frequency Estimator versus Importance Sampling. Estimated CPU time in the bottom panel is computed by fitting and extrapolating based on a quadratic function. 